\title{
SOLUTIONS IN MORREY SPACES OF SOME SEMILINEAR HEAT EQUATIONS WITH TIME-DEPENDENT EXTERNAL FORCES
}

\author{
XIAOFANG $\mathrm{ZHOU}^{\dagger}$
}

\begin{abstract}
In this paper, we consider the Cauchy problem for some semilinear heat equations with time-dependent external forces. Both the external force and the initial data are assumed to be small in some Morrey spaces. We first prove the unique existence of a small time-global solution. We next show the stability of that solution by proving the time-global sovability of perturbation problems.
\end{abstract}

\section{$\S 1$. Introduction}

In this paper, we are concerned with the existence, uniqueness and stability of solutions of the semilinear heat equations in $\mathbf{R}^{n}(n \geq 3)$ :

$$
\begin{gathered}
\frac{\partial v}{\partial t}(t, x)=\Delta v(t, x)+v(t, x)^{\nu}+f(t, x) \quad \text { in }(0, \infty) \times \mathbf{R}^{n}, \\
v(0, x)=a(x) \quad \text { on } \mathbf{R}^{n},
\end{gathered}
$$

where $\nu \geq 3, \nu \in \mathbf{Z}$.

There have been many researches on the Cauchy problem (1.1)-(1.2) without external forces, i.e. $f(t, x) \equiv 0$. (see [3]-[6] and [14]) On the other hand, many authors have also studied the Cauchy problem with measures as initial data. Brezis and Friedman [2] proved that a time-local solution exists with the Dirac measure $\delta(x)$ as the initial value if and only if $\nu<1+2 / n$. Baras and Pierre [1] studied various capacities of the initial Radon measures for which the Cauchy problem is solvable. Niwa [12] obtained a sufficient condition for the local well-posedness and the global well-posedness of the

Received April 5, 2001.

2000 Mathematics Subject Classification: Primary 35K05; Secondary 35K55, 35B35.

${ }^{\dagger}$ This research is partially supported by Resident Fellow Scheme of the United College, the Chinese University of Hong Kong. 
Cauchy problem with initial data in the spaces of measures of the Morreytype. Kozono and Yamazaki [8] obtained time-local and time-global solutions when the initial data are in the Besov-type Morrey spaces. Wu [17] concluded the well-posedness of the Cauchy problem with initial data in the homogeneous Lebesgue spaces. Then Zhou [19] showed the stability of small stationary solutions in Morrey spaces of the semilinear heat equations with time-independent external force $f(x)$.

Recently Yamazaki [18] considered the solutions in the Morrey spaces of the Navier-Stokes equation with time-dependent external force. Inspired by his research, we are interested in studying the Cauchy problem (1.1)-(1.2) when the external force $f(t, x)$ depends on $t$ and does not decay in general as $t \rightarrow \infty$. In particular, we are interested in time-periodic solutions.

The main purpose of this paper is to give a sufficient condition on the external force for the unique existence of small solutions of (1.1) in the whole time which is bounded with values in some Morrey spaces. We also show that this problem is well-posed by proving the continuous dependence of the solution on the external force. As an immediate consequence of the uniqueness, we can also prove that if the external force is time-periodic in a function space, so is the solution. The second purpose is to show the stability of small time-global solution in our functional setting.

This paper is organized as follows. In Section 2, we introduce our function spaces and state our main theorems. The existence and the uniqueness of small solutions bounded in the whole space-time are proved in Section 3. Finally, the stability of small time-global solutions is proved in Section 4.

\section{$\S 2$. Main results}

Before stating our main theorems, we will introduce the function spaces of Besov-type based on the Morrey spaces defined by H. Kozono and M. Yamazaki in [8].

Definition 2.1. Let $1 \leq q \leq p<\infty, s \in \mathbf{R}$. The Morrey space $\mathcal{M}_{p, q}$ on $\mathbf{R}^{n}$ is defined to be the set of functions $u(x) \in L_{\text {loc }}^{q}\left(\mathbf{R}^{n}\right)$ such that

$$
\|u\|_{\mathcal{M}_{p, q}}=\sup _{x_{0} \in \mathbf{R}^{n} R>0} \sup _{R} R^{n / p-n / q}\left(\int_{\left|x-x_{0}\right|<R}|u(x)|^{q} d x\right)^{1 / q}<\infty .
$$

Furthermore, the Sobolev-type Morrey space $\mathcal{M}_{p, q}^{s}$ is defined by

$$
\mathcal{M}_{p, q}^{s}=\left\{u(x) \in \mathcal{S}^{\prime} / \mathcal{P} \mid\|u(x)\|_{\mathcal{M}_{p, q}^{s}}=\left\|(-\Delta)^{\frac{s}{2}} u\right\|_{\mathcal{M}_{p, q}}<\infty\right\},
$$


where $\mathcal{S}^{\prime}$ and $\mathcal{P}$ denote the set of tempered distributions on $\mathbf{R}^{n}$ and the set of polynomials with $n$ independent vaariables, respectively.

For detailed properties, see Peetre [14], Taylor [15], Kozono and Yamazaki [8, 9], Zhou [19].

In order to define the spaces of Besov type based on the Morrey spaces, we need to use the Littlewood-Paley decomposition functions. Let $\left\{\varphi_{j}(\xi)\right\}_{j=-\infty}^{\infty}$ be a sequence of $C^{\infty}$-functions on $\mathbf{R}_{\xi}^{n}$ satisfying $0 \leq \varphi_{j}(\xi) \leq 1$, $\operatorname{supp} \varphi_{0} \subset\left\{\xi \in \mathbf{R}^{n}|1 / 2<| \xi \mid<2\right\}, \varphi_{j}(\xi)=\varphi_{0}\left(2^{-j} \xi\right)$ for every integer $j$ and every $\xi \in \mathbf{R}^{n}$, and the locally finite $\operatorname{sum} \sum_{j=-\infty}^{\infty} \varphi_{j}(\xi)$ on $\mathbf{R}_{\xi}^{n} \backslash\{0\}$ is identically equal to 1 . Then we define our function spaces as follows:

Definition 2.2. Let $1 \leq q \leq p<\infty, 1 \leq r \leq \infty$ and $s \in \mathbf{R}$. The space $\mathcal{N}_{p, q, r}^{s}=\mathcal{N}_{p, q, r}^{s}\left(\mathbf{R}^{n}\right)$ is defined to be the set of equivalence classes of distributions $u \in \mathcal{S}^{\prime} / \mathcal{P}$ such that

$$
\|u\|_{\mathcal{N}_{p, q, r}^{s}}=\left\|\left\{2^{s j}\left\|\varphi_{j}(D) u\right\|_{\mathcal{M}_{p, q}}\right\}_{j=-\infty}^{\infty}\right\|_{l^{r}}<\infty
$$

where $\varphi_{j}(D) u=\mathcal{F}^{-1}\left[\varphi_{j}(\xi) \mathcal{F}[u](\xi)\right]$, and $\mathcal{P}$ denotes the set of polynomials with $n$ variables.

The following facts are proved in [8].

Facts. The spaces $\mathcal{N}_{p, q, r}^{s}$ are Banach spaces independent of the choice of the function $\varphi_{0}(\xi)$ modulo the equivalence, and enjoy the following inclusion relations and the equivalences as Banach spaces.

1. The space $\mathcal{N}_{p, p, r}^{s}$ coincides with the standard homogeneous Besov space $\dot{B}_{p, r}^{s}$.

2. For every $s_{1}, s_{2} \in \mathbf{R}$ such that $s_{1} \neq s_{2}$, every $1 \leq q \leq p<\infty$, every $1 \leq r \leq \infty$ and every $\theta \in(0,1)$, the space $\mathcal{N}_{p, q, r}^{s_{0}(1-\theta)+s_{1} \theta}$ coincides with the real interpolation space $\left(\mathcal{M}_{p, q}^{s_{0}}, \mathcal{M}_{p, q}^{s_{1}}\right)_{\theta, r}$.

3. If $1 \leq q^{\prime}<q \leq p, 1 \leq r<r^{\prime} \leq \infty$, then we have $\mathcal{N}_{p, q, r}^{s} \subset$ $\mathcal{N}_{p, q^{\prime}, r}^{s}, \mathcal{N}_{p, q, r}^{s} \subset \mathcal{N}_{p, q, r^{\prime}}^{s}$

4. If $s<\sigma<t$, then we have $\mathcal{N}_{p, q, r}^{s} \cap \mathcal{N}_{p, q, r}^{t} \subset \mathcal{N}_{p, q, r}^{\sigma} \subset \mathcal{N}_{p, q, r}^{s}+\mathcal{N}_{p, q, r}^{t}$.

5. We have $\mathcal{N}_{p, q, 1}^{s} \subset \mathcal{M}_{p, q}^{s} \subset \mathcal{N}_{p, q, \infty}^{s}$.

6. All singular integrals are bounded on $\mathcal{M}_{p, q}^{s}$, for $1<q \leq p<\infty$ and on $\mathcal{N}_{p, q, r}^{s}$ for $1 \leq q \leq p<\infty$ and $1 \leq r \leq \infty$.

7. We have the imbedding $\mathcal{N}_{p, q, r}^{s} \subset \mathcal{N}_{\frac{p}{\theta}, \frac{q}{\theta}, r}^{s-(1-\theta) \frac{n}{p}}$ for $\theta \in(0,1)$ and $\mathcal{N}_{p, q, r}^{s} \subset \dot{B}_{\infty, r}^{s-\frac{n}{p}}$ 
8. If $s<\frac{n}{p}$, then we can choose a canonical representative from every equivalence class in $\mathcal{N}_{p, q, r}^{s}$, so that we can regard $\mathcal{N}_{p, q, r}^{s}$ as a subspace of $\mathcal{S}^{\prime}$.

9. Suppose that $1 \leq m<n$, and put $x=\left(x^{\prime}, x^{\prime \prime}\right)$, where $x^{\prime} \in \mathbf{R}^{m}$ and $x^{\prime \prime} \in \mathbf{R}^{n-m}$. Then the function $u\left(x^{\prime}\right) \in \mathcal{N}_{p, q, r}^{s}\left(\mathbf{R}^{m}\right)$, regarded as a function on $\mathbf{R}^{n}$, belongs to the space $\mathcal{N}_{\frac{n p}{m}, q, r}^{s}\left(\mathbf{R}^{n}\right)$.

10. Let $\exp (t \Delta)$ denote the heat kernel on $\mathbf{R}^{n}$. If $1 \leq q \leq p<$ $\infty, 1 \leq r \leq \infty$ and $s \in \mathbf{R}$, there exists a positive constant $C$ such that the inequality

$$
\|\exp (t \Delta) u\|_{\mathcal{N}_{p, q, r}^{s}} \leq C\|u\|_{\mathcal{N}_{p, q, r}^{s}}
$$

holds for every $t \geq 0$. Moreover, for every $p, q, r$ as above and $s<\sigma$, there exists a positive constant $C$ such that the inequality

$$
\|\exp (t \Delta) u\|_{\mathcal{N}_{p, q, 1}^{\sigma}} \leq C t^{\frac{s-\sigma}{2}}\|u\|_{\mathcal{N}_{p, q, \infty}^{s}}
$$

holds for every $t>0$.

Let $p_{0}=\frac{n(\nu-1)}{2}, p, q, r$ satisfy

$\left(\mathrm{H}_{1}\right) \quad \max \left\{\frac{\nu^{2}-\nu+1}{\nu^{2}} p_{0}, \nu\right\}<p<p_{0}, \quad \nu<r \leq p, \frac{\nu-1}{p}+\frac{1}{q}=\frac{\nu}{p_{0}}$.

The condition $\left(\mathrm{H}_{1}\right)$ implies that $\nu<p<p_{0}<q$.

For small positive constants $\varepsilon, \delta$, we define the sets

$$
\mathcal{K}=\left\{v(t, \cdot) \in L^{\infty}\left(\mathbf{R}, \mathcal{M}_{p, r} \cap \mathcal{M}_{q, \frac{q r}{p}}\right) \mid\|v\|_{\mathcal{K}}<\infty\right\}
$$

and

$$
\begin{gathered}
\mathcal{L}=\left\{f(t, \cdot) \in L^{\infty}\left(\mathbf{R}, \mathcal{N}_{p, r, \infty}^{-2-\varepsilon} \cap \mathcal{N}_{p, r, \infty}^{-2+\delta} \cap \mathcal{N}_{q, \frac{q r}{p}, \infty}^{-2-\varepsilon} \cap \mathcal{N}_{q, \frac{q r}{p}, \infty}^{-2+\delta}\right) \mid\right. \\
\left.\|f\|_{\mathcal{L}}<\infty\right\}
\end{gathered}
$$

where

$$
\begin{array}{r}
\|v\|_{\mathcal{K}}=\sup _{t \in \mathbf{R}} \max \left\{\|v(t, \cdot)\|_{\mathcal{M}_{p, r}},\|v(t, \cdot)\|_{\mathcal{M}_{q, q r}}\right\} \\
\|f\|_{\mathcal{L}}=\sup _{t \in \mathbf{R}} \max \left\{\|f(t, \cdot)\|_{\mathcal{N}_{p, r, \infty}^{-2-\varepsilon}},\|f(t, \cdot)\|_{\mathcal{N}_{p, r, \infty}^{-2+\delta}},\right. \\
\left.\left.\left\|\left.f(t, \cdot)\right|_{\mathcal{N}_{q, q r}^{-2-\varepsilon}, \infty},\right\| f(t, \cdot)\right|_{\mathcal{N}_{q, \frac{q}{p}, \infty}^{-2+\delta}}\right\} .
\end{array}
$$


We observe that the equation (1.1) formally follows from the integral equation

$$
v(t, \cdot)=\int_{-\infty}^{t} \exp ((t-\tau) \Delta)\left\{v(\tau, \cdot)^{\nu}+f(\tau, \cdot)\right\} d \tau .
$$

In general, the equation (2.1) without the initial data does not imply (1.1). However, we shall show that the small solution of (1.1) which exists on the whole space-time is uniquely determined and enjoys (2.1).

Our main result on the unique existence of small solutions of (2.1) bounded for the whole time is the following.

TheOREm 2.1. Suppose that $p, q, r$ satisfy $\left(\mathrm{H}_{1}\right)$. For small positive numbers $\varepsilon, \delta$, there exist positive numbers $\gamma_{0}$ and $\kappa_{0}$ such that the following holds.

(1) If $f(t, \cdot)$ is in $\mathcal{L}$ with $\|f\|_{\mathcal{L}}=\gamma<\gamma_{0}$, there uniquely exists a solution $v(t, x) \in \mathcal{K}$ of (2.1) such that

$$
\|v\|_{\mathcal{K}}<\kappa_{0}
$$

and $t \mapsto v(t, \cdot)$ is Hölder-continuous with values in $\mathcal{M}_{p, r} \cap \mathcal{M}_{q, \frac{q r}{p}}$.

(2) Moreover, if $t \mapsto f(t, \cdot)$ is Hölder-continuous with values in $\mathcal{N}_{p, r, \infty}^{-2} \cap$ $\mathcal{N}_{q, \frac{q r}{p}, \infty}^{-2}$, then $v(t, \cdot)$ is a unique solution of (1.1) satisfying $(2.2)$.

(3) The mapping $T$ from $B\left(0, \gamma_{0}\right) \subset \mathcal{L}$ to $B\left(0, \kappa_{0}\right) \subset \mathcal{K}$ defined by $T(f(t, \cdot))=v(t, \cdot)$ is continuous.

Remark 2.1. Suppose that $f(t, x) \in \mathcal{L}$ is periodic with respect to $t$ with period $T$, and let $v(t, x)$ be the corresponding solution. Then the function $v_{1}(t, x) \equiv v(t+T, x)$ is also the solution of (2.1) and enjoys $\left\|v_{1}\right\|_{\mathcal{K}}=\|v\|_{\mathcal{K}}$. Hence, by the uniqueness of Theorem 2.1, we see that $v_{1}(t, x) \equiv v(t, x)$, which implies that the solution $v(t, x)$ is also time-periodic with the same period $T$ as well.

Remark 2.2. Suppose that $f(t, x)$ satisfies the estimate

$$
A=\sup _{t \in \mathbf{R}} \max \left\{\|f(t, \cdot)\|_{\mathcal{N}_{\alpha, \gamma}^{-1}, \infty},\|f(t, \cdot)\|_{\mathcal{N}_{\beta, \frac{\gamma \beta}{\alpha}}^{-1}, \infty}\right\}<\infty,
$$

with some $\alpha, \beta$ and $\gamma$ such that $1<\gamma \leq \alpha<\frac{n}{2} \leq \frac{\nu-1}{\nu+1} n<\beta<n$. Then we can take some $p$ and $r$ enjoying the condition $\left(\mathrm{H}_{1}\right)$ and $\varepsilon, \delta>0$ such that 
$f(t, x) \in \mathcal{L}$ and $\|f\|_{\mathcal{L}} \leq C A$ with some positive constant $C$ independent of $f(t, x)$. In fact, we can choose $p \in\left(\beta, p_{0}\right)$ satisfying $1+\frac{n}{p}<\frac{n}{\alpha}$ and $\frac{n}{\beta}+\frac{2 p_{0}}{p}<3+\frac{n}{p_{0}}$. Putting $\varepsilon=\frac{n}{\alpha}-\frac{n}{p}-1$ and $\delta=3+\frac{n}{p_{0}}-\frac{n}{\beta}-\frac{2 p_{0}}{p}$, we see by Fact 7 that

$$
\sup _{t \in \mathbf{R}} \max \left\{\|f(t, \cdot)\|_{\mathcal{N}_{p, \frac{\gamma p}{\alpha}, \infty}^{-2-\varepsilon}},\|f(t, \cdot)\|_{\mathcal{N}_{p, \frac{\gamma p}{\alpha}, \infty}^{-1-\frac{n}{\beta}+\frac{n}{p}}}\right\} \leq C A
$$

with some positive constant $C$. Since

$$
-2+\delta, \frac{n}{p}-\frac{n}{q}-2-\varepsilon \in\left(-2-\varepsilon,-1-\frac{n}{\beta}+\frac{n}{p}\right),
$$

it follows from Fact 4 that

$$
\sup _{t \in \mathbf{R}} \max \left\{\|f(t, \cdot)\|_{\mathcal{N}_{p, \frac{\gamma p}{\alpha}, \infty}^{-2+\delta}},\|f(t, \cdot)\|_{\mathcal{N}_{p, \frac{\gamma}{\alpha}, \infty}^{\frac{n}{p}-\frac{n}{q}-2-\varepsilon}}\right\} \leq C A
$$

with another positive constant $C$. Fact 7 implies that $\mathcal{N}_{p, \frac{p}{\alpha}, \infty}^{\frac{n}{q}-\frac{n}{q}-2-\varepsilon} \subset \mathcal{N}_{q, \frac{\gamma q}{\alpha}, \infty}^{-2-\varepsilon}$ and $\mathcal{N}_{p, \frac{\gamma p}{\alpha}, \infty}^{-1-\frac{n}{\beta}+\frac{n}{p}} \subset \mathcal{N}_{q, \frac{\gamma q}{\alpha}, \infty}^{-2+\delta}$. Therefore $f(t, x) \in \mathcal{L}$ with $r=\frac{\gamma p}{\alpha}$.

EXAMPLE 2.1. We give an example of time-periodic external force enjoying the assumptions of Theorem 2.1. Let $f(t, x)=c_{0}\left(-\Delta_{x}\right)^{\frac{1}{2}}\left(1+\sin ^{2} t+\right.$ $\left.|x|^{2}\right)^{-\frac{n+1}{2}}$, then $f(t, x) \in L^{\infty}\left(\mathbf{R}, \mathcal{N}_{\alpha, \alpha, \infty}^{-1} \cap \mathcal{N}_{\beta, \beta, \infty}^{-1}\right)$ for every $\alpha, \beta$ such that $1 \leq \alpha \leq \beta$. Hence $f(t, x)$ enjoys the conditions of Remark 2.2 with some $\gamma \leq \alpha$. It follows that we can take this function as the time-periodic external force $f(t, x)$ in Theorem 2.1 provided that the constant $\left|c_{0}\right|$ is sufficiently small.

In order to state the main result on the stability of the time-global solution of (1.1), we first introduce the following condition on $p, q, r$ and $l, m, \alpha$.

$$
\begin{gathered}
\left(\mathrm{H}_{2}\right) \quad \max \left\{\frac{(\nu-1)^{2}}{\nu^{2}-\nu-1} p_{0}, \nu\right\}<p<p_{0}, \nu<r \leq p, \frac{\nu-1}{p}+\frac{1}{q}=\frac{\nu}{p_{0}}, \\
(\nu-1) p_{0} \leq l<\nu p_{0}, \quad 1<\alpha<\frac{\nu p_{0}}{l}, \quad \frac{\nu}{\alpha}<m \leq \frac{l r}{p} .
\end{gathered}
$$

It is easy to see that $p$ in the condition $\left(\mathrm{H}_{2}\right)$ also satisfies $\left(\mathrm{H}_{1}\right)$. 
Moreover, we define the set

$$
\mathcal{K}_{+}=\left\{v(t, \cdot) \in L^{\infty}\left([0, \infty), \mathcal{M}_{p, r} \cap \mathcal{M}_{q, \frac{q r}{p}}\right) \mid\|v\|_{\mathcal{K}_{+}}<\infty\right\}
$$

equipped with the norm

$$
\|v\|_{\mathcal{K}_{+}}=\sup _{t \geq 0} \max \left\{\|v(t, \cdot)\|_{\mathcal{M}_{p, r}},\|v(t, \cdot)\|_{\mathcal{M}_{q, q r},}\right\} .
$$

Let $w(t, x) \in \mathcal{K}_{+}$be a solution of (1.1) given in Theorem 2.1 with some $f(t, x)$, and let $v(t, x)$ be another solution of (1.1) with the initial data $a(x)$. Then the difference $u(t, x)=v(t, x)-w(t, x)$ enjoys the following equations:

$$
\begin{gathered}
\frac{\partial u}{\partial t}(t, x)=\Delta u(t, x)+\sum_{k=0}^{\nu-1}\left(\begin{array}{l}
\nu \\
k
\end{array}\right) u(t, x)^{\nu-k} w(t, x)^{k}, \\
u(0, x)=b(x),
\end{gathered}
$$

where $b(x)=a(x)-w(0, x)$.

For the uniqueness of solutions of the Cauchy problem (2.3)-(2.4), we have the following result.

TheOREM 2.2. Suppose that $p, q, r$ and $l, m, \alpha$ satisfy the condition $\left(\mathrm{H}_{2}\right)$. Then we can find positive numbers $\rho$ and $\lambda\left(\lambda \leq \kappa_{0}\right)$ such that the following holds. Suppose that $w(t, x) \in \mathcal{K}_{+}$is a solution of (1.1) satisfying the estimate $\|w\|_{\mathcal{K}_{+}}<\lambda$ and Hölder continuous as an $\mathcal{M}_{p, r} \cap \mathcal{M}_{q, \frac{q r}{p}}-$ valued function with respect to $t \in[0, \infty)$. Let $T$ denote either a positive constant or $\infty$ and let $b(x)$ be an element of $\mathcal{S}^{\prime}$. Then the solution of (2.3) on $(0, T) \times \mathbf{R}_{x}^{n}$ satisfying

$$
\sup _{0<t<T^{\prime}} \frac{n}{2 p_{0}}-\frac{n}{2 \alpha l}\|u(t, \cdot)\|_{\mathcal{M}_{\alpha l, \alpha m}}<1 \text { for every } T^{\prime} \in(0, T),
$$

$$
\sup _{0<t<T^{\prime}}\|u(t, \cdot)\|_{\mathcal{N}_{l, m, \infty}^{\frac{n}{p_{0}}}}^{\frac{n}{t}-\frac{n}{0}}<\infty \text { for every } T^{\prime} \in(0, T)
$$

$$
\limsup _{t \rightarrow+0} t^{\frac{n}{2 p_{0}}-\frac{n}{2 \alpha l}}\|u(t, \cdot)\|_{\mathcal{M}_{\alpha l, \alpha m}}<\rho
$$


and

$$
u(t, \cdot) \rightarrow b \text { in } \mathcal{S}^{\prime} \text { as } t \rightarrow+0
$$

is at most unique. Moreover, it is Hölder continuous as an $\mathcal{M}_{\beta l, \beta m}$-valued function with respect to $t \in(0, T)$ for every $\beta$ such that $1<\beta<\alpha$.

Remark 2.3. From the convexity inequality on $L^{p}\left(\mathbf{R}^{n}\right)$, we see that the time-global solution $w(t, x) \in \mathcal{K}_{+}$of (1.1) satisfies

$$
w(t, x) \in \mathcal{M}_{p, r} \cap \mathcal{M}_{q, \frac{q r}{p}} \subset \mathcal{M}_{p_{0}, \frac{p_{0} r}{p}} \subset \mathcal{M}_{l, \frac{l r}{p}}^{\frac{n}{l}-\frac{n}{p_{0}}} \subset \mathcal{N}_{l, m, \infty}^{\frac{n}{l}-\frac{n}{p_{0}}} .
$$

The following theorem gives the existence of time-global solution to the equation (2.3) and its asymptotic behavior. Together with Theorem 2.2, it implies the stability of the small solution given by Theorem 2.1 .

TheOrem 2.3. Let $p, q, r, l, m, \alpha$ and $w(t, x)$ be the same as in Theorem 2.2. Then there exist a positive number $\iota_{0}$ and a continuous, strictly monotone-increasing function $\omega(\iota)$ on $\left[0, \iota_{0}\right]$ with $\omega(0)=0$ such that the following holds:

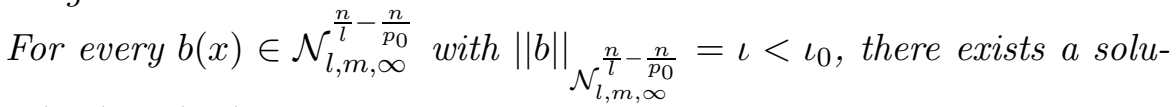
tion $u(t, x)$ of (2.3) satisfying the assumptions of Theorem 2.2 with $T=\infty$. Moreover, this solution satisfies the following conditions:

$$
\begin{gathered}
u(t, \cdot) \rightarrow \text { b weakly }{ }^{*} \text { in } \dot{B}_{\infty, \infty}^{-\frac{n}{p_{0}}} \text { as } t \rightarrow+0, \\
\sup _{t>0}\|u(t, \cdot)\|_{\mathcal{N}_{l, m, \infty}^{\frac{n}{l}-\frac{n}{p_{0}}} \leq \omega(\iota)} \leq
\end{gathered}
$$

and

(2.11) $\sup _{t>0} t^{\frac{n}{2 p_{0}}-\frac{n}{2 \beta l}}\|u(t, \cdot)\|_{\mathcal{M}_{\beta l, \beta m}}<\infty$ for every $\beta$ such that $1<\beta<\alpha$.

\section{§3. Unique existence of small solutions bounded for the whole time}

In this section we prove Theorem 2.1 by applying the functional setting introduced in the previous section. 
For functions $f(t, x), u(t, x)$ and $v(t, x)$ on $\mathbf{R}_{t} \times \mathbf{R}_{x}^{n}$, we define

$$
\begin{gathered}
\Phi_{l}[u, v](t, \cdot)=\int_{-\infty}^{t} \exp ((t-\tau) \Delta)\left\{u(\tau, \cdot)^{\nu-l} v(\tau, \cdot)^{l}\right\} d \tau, \\
l=0,1, \ldots, \nu .
\end{gathered}
$$

For $f \in \mathcal{L}$, we define the sequences of functions $\left\{v_{j}(t, x)\right\}_{j=0}^{\infty}$, $\left\{u_{j}(t, x)\right\}_{j=0}^{\infty}$ inductively by

$$
\begin{aligned}
v_{0}(t, \cdot) & =\int_{-\infty}^{t} \exp ((t-\tau) \Delta) f(\tau, \cdot) d \tau \\
v_{j+1}(t, \cdot) & =v_{0}(t, \cdot)+\int_{-\infty}^{t} \exp ((t-\tau) \Delta) v_{j}(\tau, \cdot)^{\nu} d \tau \\
& =v_{0}(t, \cdot)+\Phi_{\nu}\left[v_{j}, v_{j}\right](t, \cdot), j=0,1, \ldots, \\
u_{j}(t, \cdot) & =v_{j+1}(t, \cdot)-v_{j}(t, \cdot), j=0,1, \ldots
\end{aligned}
$$

Then we have the following lemma.

Lemma 3.1. For every s such that $-\varepsilon<s<\delta\left(<\frac{n}{q}\right)$, there exists a positive constant $C$ such that

$$
\sup _{t \in \mathbf{R}} \max \left\{\left\|v_{0}(t, \cdot)\right\|_{\mathcal{N}_{p, r, 1}^{s}},\left\|v_{0}(t, \cdot)\right\|_{\mathcal{N}_{q, q r}^{s}, 1}\right\} \leq C\|f\|_{\mathcal{L}} .
$$

In particular, we have $v_{0} \in \mathcal{K}$ and there exists a positive constant $C_{0}$ such that $\left\|v_{0}\right\|_{\mathcal{K}} \leq C_{0}\|f\|_{\mathcal{L}}$.

Proof. We use Fact 10 in the previous section to get

$$
\begin{aligned}
& \sup _{t \in \mathbf{R}} \max \left\{\left\|v_{0}(t, \cdot)\right\|_{\mathcal{N}_{p, r, 1}^{s}},\left\|v_{0}(t, \cdot)\right\|_{\mathcal{N}_{q, q r}^{s}, 1}^{s}\right\} \\
\leq & \sup _{t \in \mathbf{R}} \int_{-\infty}^{t} \max \left\{\|\exp ((t-\tau) \Delta) f(\tau, \cdot)\|_{\mathcal{N}_{p, r, 1}^{s},},\|\exp ((t-\tau) \Delta) f(\tau, \cdot)\|_{\mathcal{N}_{q, q r}^{s}, 1}\right\} d \tau \\
\leq & C \sup _{t \in \mathbf{R}}\left(\int_{-\infty}^{t-1}(t-\tau)^{-1-\frac{\varepsilon}{2}-\frac{s}{2}} d \tau+\int_{t-1}^{t}(t-\tau)^{-1+\frac{\delta}{2}-\frac{s}{2}} d \tau\right)\|f\|_{\mathcal{L}} \\
\leq & C\|f\|_{\mathcal{L}}
\end{aligned}
$$


In order to estimate the functions $v_{j}(t, x)$ and $u_{j}(t, x)$, we need the following proposition.

Proposition 3.1. Suppose that $2\left(1-\frac{p_{0}}{p}\right)<s<\frac{n}{p}-\frac{n}{p_{0}}$. Then there exists a positive constant $C_{l}$ such that the estimate

$$
\sup _{t \in \mathbf{R}} \max \left\{\left\|\Phi_{l}[u, v](t, \cdot)\right\|_{\mathcal{N}_{p, r, 1}^{s}},\left\|\Phi_{l}[u, v](t, \cdot)\right\|_{\mathcal{N}_{q, \frac{q}{p}, 1}^{s}}\right\} \leq C_{l}\|u\|_{\mathcal{K}}^{\nu-l}\|v\|_{\mathcal{K}}^{l}
$$

holds for every $u(t, x), v(t, x) \in \mathcal{K}, l=0,1, \ldots, \nu$.

Proof. Without loss of generality, let $1 \leq l \leq \nu-1$. By definition we observe that

$$
\begin{aligned}
& \left\|\Phi_{l}[u, v](t, \cdot)\right\|_{\mathcal{N}_{p, r, 1}^{s}} \\
& \leq \int_{-\infty}^{t}\left\|\exp ((t-\tau) \Delta)\left\{u(\tau, \cdot)^{\nu-l} v(\tau, \cdot)^{l}\right\}\right\|_{\mathcal{N}_{p, r, 1}^{s}} d \tau \\
& \leq C \int_{-\infty}^{t-1}(t-\tau)^{-\frac{p_{0}}{p}-\frac{s}{2}}\left\|u(\tau, \cdot)^{\nu-l} v(\tau, \cdot)^{l}\right\|_{\mathcal{N}_{p, r, \infty}^{-\frac{2 p_{0}}{p}}} d \tau \\
& +C \int_{t-1}^{t}(t-\tau)^{-1-\frac{n}{2 p_{0}}+\frac{n}{2 p}-\frac{s}{2}}\left\|u(\tau, \cdot)^{\nu-l} v(\tau, \cdot)^{l}\right\|_{\mathcal{N}_{p, r, \infty}^{-2-\frac{n}{p_{0}}+\frac{n}{p}}} d \tau, \\
& \left\|\Phi_{l}[u, v](t, \cdot)\right\|_{\mathcal{N}_{q, \frac{q r}{p}, 1}^{s}} \\
& \leq \int_{-\infty}^{t}\left\|\exp ((t-\tau) \Delta)\left\{u(\tau, \cdot)^{\nu-l} v(\tau, \cdot)^{l}\right\}\right\|_{\mathcal{N}_{q, \frac{q r}{p}, 1}^{s}} d \tau \\
& \leq C \int_{-\infty}^{t-1}(t-\tau)^{-\frac{p_{0}}{p}-\frac{s}{2}}\left\|u(\tau, \cdot)^{\nu-l} v(\tau, \cdot)^{l}\right\|_{\mathcal{N}_{q, \frac{q}{p}, \infty}^{-\frac{2 p_{0}}{p}}} d \tau \\
& +C \int_{t-1}^{t}(t-\tau)^{-\frac{p_{0}}{q}-\frac{s}{2}}\left\|u(\tau, \cdot)^{\nu-l} v(\tau, \cdot)^{l}\right\|_{\mathcal{N}_{q, \frac{q}{p}, \infty}^{-\frac{2 p_{0}}{q}}} d \tau .
\end{aligned}
$$

Next, we apply the Hölder inequality and embedding theorems on Morrey 
spaces to get (see Lemma 2.3, Lemma 2.4 of [19], together with Facts 5,7)

$$
\begin{aligned}
& \left\|u(\tau, \cdot)^{\nu-l} v(\tau, \cdot)^{l}\right\|_{\mathcal{N}_{p, r, \infty}^{-}}^{-\frac{2 p_{0}}{p}} \leq C\left\|u(\tau, \cdot)^{\nu-l} v(\tau, \cdot)^{l}\right\|_{\mathcal{N}_{\frac{p}{\nu}, \frac{r}{\nu}, \infty}^{0}} \\
& \leq C\left\|u(\tau, \cdot)^{\nu-l}\right\|_{\mathcal{M}_{\frac{p}{\nu-l}, \frac{r}{\nu-l}}}\left\|v(\tau, \cdot)^{l}\right\|_{\mathcal{M}_{\frac{p}{\tau}, \frac{r}{\tau}}} \\
& \leq C\|u(\tau, \cdot)\|_{\mathcal{M}_{p, r}}^{\nu-l}\|v(\tau, \cdot)\|_{\mathcal{M}_{p, r}}^{l} \\
& \leq C\|u\|_{\mathcal{K}}^{\nu-l}\|v\|_{\mathcal{K}}^{l}
\end{aligned}
$$

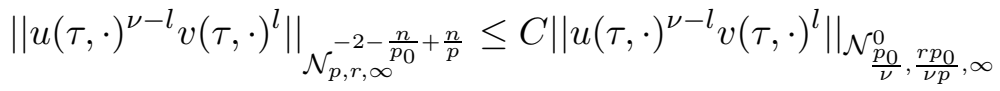

$$
\begin{aligned}
& \leq C\left\|u(\tau, \cdot)^{\nu-l} v(\tau, \cdot)^{l}\right\|_{\mathcal{M}_{\frac{p_{0}}{\nu}, \frac{r p_{0}}{\nu p}}}
\end{aligned}
$$

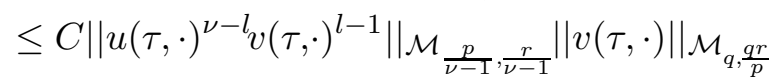

$$
\begin{aligned}
& \leq C\|u(\tau, \cdot)\|_{\mathcal{M}_{p, r}}^{\nu-l}\|v(\tau, \cdot)\|_{\mathcal{M}_{p, r}}^{l-1}\|v(\tau, \cdot)\|_{\mathcal{M}_{q, \frac{q r}{p}}} \\
& \leq C\|u\|_{\mathcal{K}}^{\nu-l}\|v\|_{\mathcal{K}}^{l} \\
& \left\|u(\tau, \cdot)^{\nu-l} v(\tau, \cdot)^{l}\right\|_{\mathcal{N}_{q, \frac{q r}{p}, \infty}^{-\frac{2 p_{0}}{p}}} \leq C\left\|u(\tau, \cdot)^{\nu-l} v(\tau, \cdot)^{l}\right\|_{\mathcal{N}_{p, r, \infty}^{-2-\frac{n}{p_{0}}+\frac{n}{p}}}, \\
& \left\|u(\tau, \cdot)^{\nu-l} v(\tau, \cdot)^{l}\right\|_{\mathcal{N}_{q, \frac{q r}{p}, \infty}^{-\frac{2 p_{0}}{q}}} \leq C\left\|u(\tau, \cdot)^{\nu-l} v(\tau, \cdot)^{l}\right\|_{\mathcal{N}_{\frac{q}{\nu}, \frac{q r}{p \nu}, \infty}^{0}} \\
& \leq C\|u(\tau, \cdot)\|_{\mathcal{M}_{q, \frac{q r}{p}}^{\nu-l}}^{\nu}\|v(\tau, \cdot)\|_{\mathcal{M}_{q, \frac{q r}{p}}}^{l} \\
& \leq C\|u\|_{\mathcal{K}}^{\nu-l}\|v\|_{\mathcal{K}}^{l} \text {. }
\end{aligned}
$$

Hence we get the following estimates:

$$
\begin{aligned}
& \left\|\Phi_{l}[u, v](t, \cdot)\right\|_{\mathcal{N}_{p, r, 1}^{s}} \\
\leq & C\left\{\int_{-\infty}^{t-1}(t-\tau)^{-\frac{p_{0}}{p}-\frac{s}{2}} d \tau+\int_{t-1}^{t}(t-\tau)^{-1-\frac{n}{2 p_{0}}+\frac{n}{2 p}-\frac{s}{2}} d \tau\right\}\|u\|_{\mathcal{K}}^{\nu-l}\|v\|_{\mathcal{K}}^{l} \\
\leq & C\|u\|_{\mathcal{K}}^{\nu-l}\|v\|_{\mathcal{K}}^{l}, \\
& \left\|\Phi_{l}[u, v](t, \cdot)\right\|_{\mathcal{N}_{q, \frac{q}{p}}^{s}, 1} \\
\leq & C\left\{\int_{-\infty}^{t-1}(t-\tau)^{-\frac{p_{0}}{p}-\frac{s}{2}} d \tau+\int_{t-1}^{t}(t-\tau)^{-\frac{p_{0}}{q}-\frac{s}{2}} d \tau\right\}\|u\|_{\mathcal{K}}^{\nu-l}\|v\|_{\mathcal{K}}^{l} \\
\leq & C\|u\|_{\mathcal{K}}^{\nu-l}\|v\|_{\mathcal{K}}^{l},
\end{aligned}
$$

which implies the proposition. 
COROLlary 3.1. There exist positive constants $C_{2, l}$ such that

$$
\left.\left\|\Phi_{l}[u, v]\right\|\right|_{\mathcal{K}} \leq C_{2, l}\|u\|_{\mathcal{K}}^{\nu-l}\|v\|_{\mathcal{K}}^{l}
$$

for every $u(t, x), v(t, x) \in \mathcal{K}, l=0,1, \ldots, \nu$.

By virtue of Corollary 3.1 we can prove the next lemma.

LEMmA 3.2. We have $v_{j}, u_{j} \in \mathcal{K}$ for every $j=0,1, \ldots$, and the estimates

$$
\left\|u_{0}\right\|_{\mathcal{K}} \leq C_{2, \nu}\left\|v_{0}\right\|_{\mathcal{K}}^{\nu}, \quad\left\|u_{j}\right\|_{\mathcal{K}} \leq \sum_{l=0}^{\nu-1}\left(\begin{array}{l}
\nu \\
l
\end{array}\right) C_{2, l}\left\|u_{j-1}\right\|_{\mathcal{K}}^{\nu-l}\left\|v_{j-1}\right\|_{\mathcal{K}}^{l}
$$

hold for every $j=1,2, \ldots$

Proof. We proceed by induction on $j$. Since $u_{0}=v_{1}-v_{0}=\Phi_{\nu}\left[v_{0}, v_{0}\right](t, \cdot)$, the statement for $v_{1}, u_{0}$ follows immediately from Lemma 3.1 and Corollary 3.1. Assume that the assertion has been proved for $j \geq 1$. We see that

$$
\begin{aligned}
u_{j}=v_{j+1}-v_{j} & =\Phi_{\nu}\left[v_{j}, v_{j}\right]-\Phi_{\nu}\left[v_{j-1}, v_{j-1}\right] \\
& =\sum_{l=0}^{\nu-1}\left(\begin{array}{l}
\nu \\
l
\end{array}\right) \Phi_{l}\left[u_{j-1}, v_{j-1}\right] .
\end{aligned}
$$

Corollary 3.1 implies that $u_{j} \in \mathcal{K}$ and

$$
\left\|u_{j}\right\|_{\mathcal{K}} \leq \sum_{l=0}^{\nu-1}\left(\begin{array}{l}
\nu \\
l
\end{array}\right) C_{2, l}\left\|u_{j-1}\right\|_{\mathcal{K}}^{\nu-l}\left\|v_{j-1}\right\|_{\mathcal{K}}^{l}
$$

Since $v_{j+1}=v_{j}+u_{j}$, the fact $v_{j+1} \in \mathcal{K}$ follows from the induction hypothesis.

We now begin the proof of Theorem 2.1 with $\gamma_{0}=\frac{1}{4 C_{0} C_{2}}, \kappa_{0}=\frac{1}{2 C_{2}}$, where $C_{0}$ is the constant in Lemma 3.1 and $C_{2}=\sum_{l=0}^{\nu}\left(\begin{array}{l}\nu \\ l\end{array}\right) C_{2, l} 2^{\nu}$. Without loss of generality, let $C_{0}, C_{2}>1$.

First we define

$$
A_{j}=\left\|v_{j}\right\|_{\mathcal{K}}, \quad B_{j}=\left\|u_{j}\right\|_{\mathcal{K},}, \quad j=0,1, \ldots
$$

Then we have the following estimates. 
Lemma 3.3. There exist positive constants $C_{0}, C_{2}$ such that

$$
A_{j+1} \leq C_{0} \gamma+C_{2} A_{j}^{2}
$$

for every $j=0,1, \ldots$

Proof. From Lemma 3.1 and Corollary 3.1, we have

$$
\begin{aligned}
\left\|v_{j+1}\right\|_{\mathcal{K}} & =\left.\left\|v_{0}+\Phi_{\nu}\left[v_{j}, v_{j}\right]\right\|\right|_{\mathcal{K}} \leq\left\|v_{0}\right\|_{\mathcal{K}}+\left\|\Phi_{\nu}\left[v_{j}, v_{j}\right]\right\|_{\mathcal{K}} \\
& \leq C_{0}\|f\|_{\mathcal{L}}+C_{2, \nu}\left\|v_{j}\right\|_{\mathcal{K}}^{\nu} \leq C_{0} \gamma+C_{2}\left\|v_{j}\right\|_{\mathcal{K}}^{2},
\end{aligned}
$$

which completes the proof. The constant $C_{2}=\sum_{l=0}^{\nu} C_{2, l} 2^{\nu}$.

Let $\gamma_{0}=\frac{1}{4 C_{0} C_{2}}, \kappa_{0}=\frac{1}{2 C_{2}}$. From Lemma 3.3 we see that if $\gamma<\gamma_{0}$, then we have

$$
A_{j} \leq \psi(\gamma)=\frac{1-\sqrt{1-4 C_{0} C_{2} \gamma}}{2 C_{2}}<\kappa_{0}=\psi\left(\gamma_{0}\right)=\frac{1}{2 C_{2}}<1
$$

for every $j=1,2, \ldots$, by induction on $j$.

From Lemma 3.2 we obtain

$$
\begin{aligned}
B_{j}=\left\|u_{j}\right\|_{\mathcal{K}} & \leq \sum_{l=0}^{\nu-1}\left(\begin{array}{l}
\nu \\
l
\end{array}\right) C_{2, l}|| u_{j-1}\left\|_{\mathcal{K}}^{\nu-l}\right\| v_{j-1} \|_{\mathcal{K}}^{l} \\
& \leq \sum_{l=0}^{\nu-1}\left(\begin{array}{l}
\nu \\
l
\end{array}\right) C_{2, l}\left(\left\|v_{j}\right\|_{\mathcal{K}}+\left\|v_{j-1}\right\|_{\mathcal{K}}\right)^{\nu-l-1}\left\|v_{j-1}\right\|_{\mathcal{K}}^{l}\left\|u_{j-1}\right\|_{\mathcal{K}} \\
& \leq \sum_{l=0}^{\nu-1}\left(\begin{array}{l}
\nu \\
l
\end{array}\right) C_{2, l} 2^{\nu} \psi(\gamma)^{\nu-1} B_{j-1} \\
& \leq C_{2} \psi(\gamma) B_{j-1}<C_{2} \psi\left(\gamma_{0}\right) B_{j-1}=\frac{1}{2} B_{j-1}
\end{aligned}
$$

for every $j=1,2, \ldots$, which implies that $\sum_{j=0}^{\infty} B_{j}<\infty$. It follows that $\sum_{j=0}^{k} u_{j}(t, \cdot)$ converges in $\mathcal{M}_{p, r} \cap \mathcal{M}_{q, \frac{q r}{p}}$ as $k \rightarrow \infty$ for almost every $t \in \mathbf{R}$, and that there exists a function $v(t, \cdot) \in \mathcal{K}$ such that $v_{0}(t, \cdot)+\sum_{j=0}^{\infty} u_{j}(t, \cdot)=v(t, \cdot)$ for almost every $t>0$. Moreover, we have the estimate

$$
\|v\|_{\mathcal{K}} \leq \frac{1-\sqrt{1-4 C_{0} C_{2} \gamma}}{2 C_{2}}<\kappa_{0}=\psi\left(\gamma_{0}\right)=\frac{1}{2 C_{2}}
$$


Hence from the formula $v_{j+1}(t, \cdot)=v_{0}(t, \cdot)+\Phi_{\nu}\left[v_{j}, v_{j}\right](t, \cdot)$ it follows that $v$ satisfies the integral equation (2.1). This completes the proof of the existence and the required sharper estimate (2.2) in Theorem 2.1 .

Next we will show the uniqueness of the solutions of (2.1). Let $u(t, x)$ be another solution of (2.1) such that $\|u\|_{\mathcal{K}}<\kappa_{0}$. Putting $w(t, x)=u(t, x)-v(t, x)$, we have

$$
w(t, \cdot)=\sum_{l=0}^{\nu-1}\left(\begin{array}{l}
\nu \\
l
\end{array}\right) \Phi_{l}[w, v](t, \cdot) .
$$

Corollary 3.1 implies that

$$
\begin{aligned}
\|w\|_{\mathcal{K}} & \leq \sum_{l=0}^{\nu-1}\left(\begin{array}{l}
\nu \\
l
\end{array}\right) C_{2, l}\|w\|_{\mathcal{K}}^{\nu-l}\|v\|_{\mathcal{K}}^{l} \leq \sum_{l=0}^{\nu-1}\left(\begin{array}{l}
\nu \\
l
\end{array}\right) C_{2, l} 2^{\nu} \kappa_{0}{ }^{\nu-1}\|w\|_{\mathcal{K}} \\
& \leq C_{2} \kappa_{0}\|w\|_{\mathcal{K}}=\frac{1}{2}\|w\|_{\mathcal{K}},
\end{aligned}
$$

we have $\|w\|_{\mathcal{K}}=0$, which implies $u \equiv v$. This proves the uniqueness of the solutions of (2.1).

Lemma 3.4. $v(t, x)$ is Hölder-continuous in $t$ with values in $\mathcal{M}_{p, r} \cap$ $\mathcal{M}_{q, \frac{q r}{p}}$.

Proof. For every $s, t$ such that $s<t \leq s+1$, we have

$$
v(t, \cdot)-v(s, \cdot)=\mathbf{I}_{1}+\mathbf{I}_{2}+\mathbf{I}_{3},
$$

where

$$
\begin{aligned}
& \mathbf{I}_{1}=\int_{s}^{t} \exp ((t-\tau) \Delta)\left\{v(\tau, \cdot)^{\nu}\right\} d \tau, \\
& \mathbf{I}_{2}=\int_{s}^{t} \exp ((t-\tau) \Delta) f(\tau, \cdot) d \tau, \\
& \mathbf{I}_{3}=(\exp ((t-s) \Delta)-1) v(s, \cdot) .
\end{aligned}
$$

First we consider $\|v(t, \cdot)-v(s, \cdot)\|_{\mathcal{M}_{p, r}}$. Denote $\sigma_{1}=\frac{n \nu}{q}-\frac{n}{p}$, then we 
have $0<\sigma_{1}<2$ and

$$
\begin{aligned}
& \left\|\mathbf{I}_{1}\right\|_{\mathcal{M}_{p, r}} \leq C \int_{s}^{t}\left\|\exp ((t-\tau) \Delta)\left\{v(\tau, \cdot)^{\nu}\right\}\right\|_{\mathcal{N}_{p, r, 1}^{0}} d \tau \\
& \leq C \int_{s}^{t}\left\|\exp ((t-\tau) \Delta)\left\{v(\tau, \cdot)^{\nu}\right\}\right\|_{\mathcal{N}_{\frac{q}{\nu}, \frac{q r}{p \nu}, 1}^{\sigma_{1}}} d \tau \\
& \leq C \int_{s}^{t}(t-\tau)^{-\frac{\sigma_{1}}{2}}\left\|\left\{v(\tau, \cdot)^{\nu}\right\}\right\|_{\mathcal{N}_{\frac{q}{\nu}, \frac{q r}{p \nu}, \infty}^{0}} d \tau \\
& \leq C \int_{s}^{t}(t-\tau)^{-\frac{\sigma_{1}}{2}}\|v(\tau, \cdot)\|_{\mathcal{M}_{q, \frac{q}{p}}}^{\nu} d \tau \\
& \leq C(t-s)^{1-\frac{\sigma_{1}}{2}}\|v(\tau, \cdot)\|_{\mathcal{M}_{q, \frac{q r}{p}}}^{\nu} \leq C(t-s)^{1-\frac{\sigma_{1}}{2}} \text {, } \\
& \left\|\mathbf{I}_{2}\right\|_{\mathcal{M}_{p, r}} \leq \int_{s}^{t}\|\exp ((t-\tau) \Delta) f(\tau, \cdot)\|_{\mathcal{N}_{p, r, 1}^{0}} d \tau \\
& \leq C \int_{s}^{t}(t-\tau)^{-1+\frac{\delta}{2}}\|f(\tau, \cdot)\|_{\mathcal{N}_{p, r, \infty}^{-2+\delta}} d \tau \leq C(t-s)^{\frac{\delta}{2}}\|f\|_{\mathcal{L}} .
\end{aligned}
$$

On the other hand, choosing $\sigma_{2}$ so that $0<\sigma_{2}<\min \left\{\delta, \frac{n}{p}-\frac{n}{p_{0}}\right\}$, we apply Lemma 3.1 and Proposition 3.1 to get

$$
\begin{aligned}
\left\|\mathbf{I}_{3}\right\|_{\mathcal{M}_{p, r}} & \leq \int_{s}^{t}\left\|\frac{d}{d \tau} \exp ((\tau-s) \Delta) v(s, \cdot)\right\|_{\mathcal{M}_{p, r}} d \tau \\
& \leq C \int_{s}^{t}(\tau-s)^{-1+\frac{\sigma_{2}}{2}}\|v(s, \cdot)\|_{\mathcal{N}_{p, r, 1}^{\sigma_{2}}} d \tau \\
& \leq C(t-s)^{\frac{\sigma_{2}}{2}}\left(\|f\|_{\mathcal{L}}+\|v\|_{\mathcal{K}}^{\nu}\right) \leq C(t-s)^{\frac{\sigma_{2}}{2}} .
\end{aligned}
$$

In view of the inequalities above, we conclude that

$$
\|v(t, \cdot)-v(s, \cdot)\|_{\mathcal{M}_{p, r}} \leq C\left\{(t-s)^{\frac{\sigma_{2}}{2}}+(t-s)^{1-\frac{\sigma_{1}}{2}}\right\}
$$


Next we consider $\|v(t, \cdot)-v(s, \cdot)\|_{\mathcal{M}_{q, \frac{q r}{p}}}$.

$$
\begin{aligned}
& \left\|\mathbf{I}_{1}\right\|_{\mathcal{M}_{q, \frac{q r}{p}}} \leq C \int_{s}^{t}\left\|\exp ((t-\tau) \Delta)\left\{v(\tau, \cdot)^{\nu}\right\}\right\|_{\mathcal{N}_{q, \frac{q r}{p}, 1}^{0}} d \tau
\end{aligned}
$$

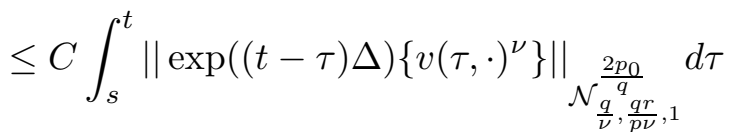

$$
\begin{aligned}
& \leq C \int_{s}^{t}(t-\tau)^{-\frac{p_{0}}{q}}\|v(\tau, \cdot)\|_{\mathcal{M}_{q, \frac{q}{p}}}^{\nu} d \tau \\
& \leq C(t-s)^{1-\frac{p_{0}}{q}}\|v\|_{\mathcal{K} .}^{\nu}
\end{aligned}
$$

Moreover, it is easy to obtain the following estimates in the same way as in (3.1).

$$
\begin{aligned}
& \left\|\mathbf{I}_{2}\right\|_{\mathcal{M}_{q, \frac{q r}{p}}} \leq C(t-s)^{\frac{\delta}{2}}\|f\|_{\mathcal{L}}, \\
& \left\|\mathbf{I}_{3}\right\|_{\mathcal{M}_{q, \frac{q}{p}}} \leq C \int_{s}^{t}\left\|\frac{d}{d \tau} \exp ((\tau-s) \Delta) v(s, \cdot)\right\|_{\mathcal{M}_{q, \frac{q r}{p}}} d \tau \\
& \leq C \int_{s}^{t}(\tau-s)^{-1+\frac{\sigma_{2}}{2}}\|v(s, \cdot)\|_{\mathcal{N}_{q, \frac{q r}{p}, 1}^{\sigma_{2}}} d \tau \\
& \leq C(t-s)^{\frac{\sigma_{2}}{2}}\left(\|f\|_{\mathcal{L}}+\|v\|_{\mathcal{K}}^{\nu}\right) \leq C(t-s)^{\frac{\sigma_{2}}{2}} .
\end{aligned}
$$

Hence we have

$$
\|v(t, \cdot)-v(s, \cdot)\|_{\mathcal{M}_{q, \frac{q r}{p}}} \leq\left\{(t-s)^{\frac{\sigma_{2}}{2}}+(t-s)^{1-\frac{p_{0}}{q}}\right\} .
$$

By (3.1) and (3.2), $v(t, x)$ is Hölder-continuous with values in $\mathcal{M}_{p, r} \cap$ $\mathcal{M}_{q, \frac{q r}{p}}$

Proposition 3.2. If $f(t, \cdot)$ is Hölder-continuous with values in $\mathcal{N}_{p, r, \infty}^{-2} \cap \mathcal{N}_{q, \frac{q r}{p}, \infty}^{-2}$, then the solution $v(t, x) \in \mathcal{K}$ of $(2.1)$ also solves the equation (1.1).

Proof. It is enough to show the required Hölder continuity of the function $\Gamma(t, \cdot) \equiv v(t, \cdot)^{\nu}+f(t, \cdot)$. Then we can complete the proof in the same way as in Theorem 5.1 of [19].

By Lemma 3.4 there exists a positive number $\theta$ such that

$$
\|v(t, \cdot)-v(s, \cdot)\|_{\mathcal{M}_{p, r}} \leq C(t-s)^{\theta}, \quad\|v(t, \cdot)-v(s, \cdot)\|_{\mathcal{M}_{q, \frac{q r}{p}}} \leq C(t-s)^{\theta}
$$


for every $s, t$ such that $s<t \leq s+1$.

Therefore

$$
\begin{aligned}
& \left\|v(t, \cdot)^{\nu}-v(s, \cdot)^{\nu}\right\|_{\mathcal{N}_{p, r, \infty}^{-2}} \\
& \leq C \sum_{l=0}^{\nu-1}\left(\begin{array}{l}
\nu \\
l
\end{array}\right)\left\|(v(t, \cdot)-v(s, \cdot))^{\nu-l} v(s, \cdot)^{l}\right\|_{\mathcal{N}_{\frac{n p}{n+2 p}, \frac{n r}{n+2 p}, \infty}^{0}} \\
& \leq C \sum_{l=0}^{\nu-1}\left(\begin{array}{l}
\nu \\
l
\end{array}\right)\left\|(v(t, \cdot)-v(s, \cdot))^{\nu-l} v(s, \cdot)^{l}\right\|_{\mathcal{M}_{\frac{n p}{n+2 p}, \frac{n r}{n+2 p}}} \\
& \leq C \sum_{l=0}^{\nu-1}\left(\begin{array}{l}
\nu \\
l
\end{array}\right)\|v(t, \cdot)-v(s, \cdot)\|_{\mathcal{M}_{p, r}}\left\|(v(t, \cdot)-v(s, \cdot))^{\nu-1-l} v(s, \cdot)^{l}\right\|_{\mathcal{M}_{\frac{p_{0}}{\nu-1}, \frac{p_{0} r}{(\nu-1) p}}} \\
& \leq C \sum_{l=0}^{\nu-1}\left(\begin{array}{l}
\nu \\
l
\end{array}\right)(t-s)^{\theta}\|v(t, \cdot)-v(s, \cdot)\|_{\mathcal{M}_{p_{0}, \frac{p_{0} r}{p}}^{\nu-1-l}}\|v(s, \cdot)\|_{\mathcal{M}_{p_{0}, \frac{p_{0} r}{p}}}^{l} \\
& \leq C(t-s)^{\theta} \text {, } \\
& \left\|v(t, \cdot)^{\nu}-v(s, \cdot)^{\nu}\right\|_{\mathcal{N}_{q, \frac{q r}{p}, \infty}^{-2}} \\
& \leq C \sum_{l=0}^{\nu-1}\left(\begin{array}{l}
\nu \\
l
\end{array}\right)\left\|(v(t, \cdot)-v(s, \cdot))^{\nu-l} v(s, \cdot)^{l}\right\|_{\mathcal{N}_{n q}^{0}{ }_{n+2 q}, \frac{n q r}{(n+2 q) p}, \infty} \\
& \leq C \sum_{l=0}^{\nu-1}\left(\begin{array}{l}
\nu \\
l
\end{array}\right)\left\|(v(t, \cdot)-v(s, \cdot))^{\nu-l} v(s, \cdot)^{l}\right\|_{\mathcal{M}_{\frac{n q}{n+2 q}, \frac{n q r}{(n+2 q) p}}} \\
& \leq C \sum_{l=0}^{\nu-1}\left(\begin{array}{l}
\nu \\
l
\end{array}\right)\|v(t, \cdot)-v(s, \cdot)\|_{\mathcal{M}_{q, \frac{q r}{p}}} \\
& \times\left\|(v(t, \cdot)-v(s, \cdot))^{\nu-1-l} v(s, \cdot)^{l}\right\|_{\mathcal{M}_{\frac{p_{0}}{\nu-1}, \frac{p_{0} r}{(\nu-1) p}}} \\
& \leq C \sum_{l=0}^{\nu-1}\left(\begin{array}{l}
\nu \\
l
\end{array}\right)(t-s)^{\theta}\|v(t, \cdot)-v(s, \cdot)\|_{\mathcal{M}_{p_{0}, \frac{p_{0} r}{p}}^{\nu-1-l}}\|v(s, \cdot)\|_{\mathcal{M}_{p_{0}, \frac{p_{0} r}{p}}}^{l} \\
& \leq C(t-s)^{\theta} \text {, }
\end{aligned}
$$

which complete the proof.

In order to prove the uniqueness of the solution of (1.1), we need the following proposition.

Proposition 3.3. Assume that the solution $v(t, x) \in \mathcal{K}$ of $(1.1)$ enjoys 
the estimate (2.2) and is Hölder-continuous in t with values in $\mathcal{M}_{p, r} \cap \mathcal{M}_{q, \frac{q r}{p}}$. Then $v(t, x)$ solves $(2.1)$.

Proof. Put $u(t, x)=v_{0}(t, x)+\Phi_{\nu}[v, v](t, x)$. Then Lemma 3.1 and Corollary 3.1 implies that $u(t, x) \in \mathcal{K}$. Moreover, the Hölder continuity of $v(t, x)$ implies that of $\Phi_{\nu}[v, v](t, x)$. Hence $u(t, x)$ satisfies the differential equation

$$
\frac{\partial u}{\partial t}=\Delta u+v^{\nu}+f
$$

Denote $w(t, x)=u(t, x)-v(t, x)$, then $w(t, x) \in \mathcal{K}$ and

$$
\frac{\partial w}{\partial t}=\Delta w
$$

Hence, for every $s, t \in \mathbf{R}$ such that $s<t$, we have the estimate

$$
\begin{aligned}
\|w(t, \cdot)\|_{\mathcal{M}_{q, \frac{q r}{p}}} & \leq C\|w(t, \cdot)\|_{\mathcal{N}_{q, \frac{q}{p}, 1}^{0}} \leq C\|w(t, \cdot)\|_{\mathcal{N}_{p, r, 1}^{\frac{n}{p}-\frac{n}{q}}} \\
& \leq C(t-s)^{\frac{n}{2 q}-\frac{n}{2 p}}\|w(s, \cdot)\|_{\mathcal{N}_{p, r, \infty}^{0}} \\
& \leq C(t-s)^{\frac{n}{2 q}-\frac{n}{2 p}}\|w(s, \cdot)\|_{\mathcal{M}_{p, r} .}
\end{aligned}
$$

Since $\|w(s, \cdot)\|_{\mathcal{M}_{p, r}}$ is bounded from above, we see that the right-hand side of the formula above tends to 0 as $s \rightarrow-\infty$ for every fixed $t \in \mathbf{R}$. Hence $\|w(t, \cdot)\|_{\mathcal{M}_{q, q r} \frac{}{p}}=0$ for every $t \in \mathbf{R}$, which yields $w \equiv 0$. Hence $v \equiv u=$ $v_{0}(t, x)+\Phi_{\nu}[v, v](t, x)$. It follows that $v(t, x)$ solves $(2.1)$.

Remark 3.1. Proposition 3.3 and the uniqueness of the solutions of (2.1) imply the uniqueness of the solutions of (1.1).

Finally, it remains only to show the continuity of the mapping $T: f(t, x) \mapsto v(t, x)$ to prove Theorem 2.1. Suppose that $v(t, x)$ is the unique solution of $(2.1)$ with $\|v\|_{\mathcal{K}}<\kappa_{0}$ for $f \in \mathcal{L}$ such that $\|f\|_{\mathcal{L}}<\gamma_{0}$, and that $u(t, x)$ is the unique solution of (2.1) with $\|u\|_{\mathcal{K}}<\kappa_{0}$ with $f(t, x)$ replaced by $g(t, x) \in \mathcal{L}$ such that $\|g\|_{\mathcal{L}}<\gamma_{0}$.

Since

$$
\begin{aligned}
u-v & =u_{0}+\Phi_{\nu}[u, u]-\left(v_{0}+\Phi_{\nu}[v, v]\right) \\
& =\left(u_{0}-v_{0}\right)+\sum_{l=0}^{\nu-1}\left(\begin{array}{l}
\nu \\
l
\end{array}\right) \Phi_{l}[u-v, v],
\end{aligned}
$$


Lemma 3.1 and Corollary 3.1 imply that

$$
\begin{aligned}
\left\|u_{0}-v_{0}\right\|_{\mathcal{K}} & \leq C_{0}\|f-g\|_{\mathcal{L}} \\
\left\|\sum_{l=0}^{\nu-1}\left(\begin{array}{l}
\nu \\
l
\end{array}\right) \Phi_{l}[u-v, v]\right\|_{\mathcal{K}} & \\
& \leq\left.\sum_{l=0}^{\nu-1}\left(\begin{array}{l}
\nu \\
l
\end{array}\right)\left\|\Phi_{l}[u-v, v]\right\|\right|_{\mathcal{K}} \\
& \leq \sum_{l=0}^{\nu-1}\left(\begin{array}{l}
\nu \\
l
\end{array}\right) C_{2, l}\left(\|u\|_{\mathcal{K}}+\|v\|_{\mathcal{K}}\right)^{\nu-1-l}\|v\|_{\mathcal{K}}^{l}\|u-v\|_{\mathcal{K}} \\
& \leq \sum_{l=0}^{\nu-1}\left(\begin{array}{l}
\nu \\
l
\end{array}\right) C_{2, l} 2^{\nu} \kappa_{0}{ }^{\nu-1}\|u-v\|_{\mathcal{K}}=\frac{1}{2} \kappa_{0}{ }^{\nu-2}\|u-v\|_{\mathcal{K}} .
\end{aligned}
$$

It follows that

$$
\|u-v\|_{\mathcal{K}} \leq \frac{2 C_{0}}{2-\kappa_{0}{ }^{\nu-2}}\|f-g\|_{\mathcal{L}}
$$

which proves the continuity of the mapping $T: f(t, x) \mapsto v(t, x)$.

Thus we have completely proved Theorem 2.1.

\section{$\S 4$. Stability of time-global solutions}

In this section we prove the stability of the time-global solutions of (1.1) given in Theorem 2.1 by applying the successive approximation. Since the solution $w(t, x)$ depends on $t$, it is hard to apply the perturbation theory of semigroups as in [19]. But $w(t, x)$ has both better decay property and better smoothness than the general elements of $\mathcal{M}_{p_{0}, \frac{p_{0} r}{p}}$, so we can use the heat semigroup to control the perturbations.

In the sequel we always assume that $p, q, r$ and $l, m, \alpha$ satisfy the condition $\left(\mathrm{H}_{2}\right)$, and $w(t, x) \in \mathcal{K}_{+}$is a solution of (1.1) with $\|w\|_{\mathcal{K}_{+}}<1$ given in Theorem 2.1. Let $T$ be either a positive number or $\infty$. We introduce the set

$$
\mathcal{J}_{\alpha, T}=\left\{\left.u(t, x)\left|\sup _{0<t<T} t^{\frac{n}{2 p_{0}}-\frac{n}{2 \alpha l}} \| u(t, \cdot)\right|\right|_{\mathcal{M}_{\alpha l, \alpha m}}<\infty\right\}
$$


equipped with the norm

$$
\|u\|_{\mathcal{J}_{\alpha, T}}=\sup _{0<t<T} t^{\frac{n}{2 p_{0}}-\frac{n}{2 \alpha l}}\|v(t, \cdot)\|_{\mathcal{M}_{\alpha l, \alpha m}} .
$$

The integral equation corresponding to (2.3)-(2.4) is as follows.

$$
u(t, x)=\exp (t \Delta) b(x)+\int_{0}^{t} \exp ((t-\tau) \Delta) \sum_{k=0}^{\nu-1}\left(\begin{array}{l}
\nu \\
k
\end{array}\right) u(\tau, \cdot)^{\nu-k} w(\tau, \cdot)^{k} d \tau .
$$

We also define

$$
\Psi[u](t, \cdot)=\int_{0}^{t} \exp ((t-\tau) \Delta) \sum_{k=0}^{\nu-1}\left(\begin{array}{l}
\nu \\
k
\end{array}\right) u(\tau, \cdot)^{\nu-k} w(\tau, \cdot)^{k} d \tau .
$$

Then we have the following proposition.

Proposition 4.1. For every $\sigma$ such that $\frac{n}{l}-\frac{n \nu}{\alpha l}<\sigma \leq \frac{n}{l}-\frac{n}{\alpha l}$, there exists a positive constant $C$ independent of $w \in \mathcal{K}_{+}$and $T \in(0, \infty]$ such that the estimate

$$
\begin{aligned}
& \|\Psi[u]-\Psi[v]\|_{\mathcal{N}_{l, m, 1}^{\sigma}} \\
\leq & C \sum_{k=1}^{\nu-1} \sum_{j=0}^{\nu-k-1} C_{\nu, k, j}\left(\|u\|_{\mathcal{J}_{\alpha, T}}+\|v\|_{\mathcal{J}_{\alpha, T}}\right)^{\nu-k-1-j}\|v\|_{\mathcal{J}_{\alpha, T}}^{j}\|w\|_{\mathcal{K}_{+}}^{k} \\
& \int_{0}^{s}(t-\tau)^{\frac{n}{2 l}-\frac{n(\nu-k)}{2 \alpha l}-\frac{n k}{2 p}-\frac{\sigma}{2}} \tau^{\left(\frac{n}{2 \alpha l}-\frac{n}{2 p_{0}}\right)(\nu-k-1)}\|u(\tau, \cdot)-v(\tau, \cdot)\|_{\mathcal{M}_{\alpha l, \alpha m}} d \tau \\
+ & C \sum_{k=1}^{\nu-1} \sum_{j=0}^{\nu-k-1} C_{\nu, k, j}\left(\|u\|_{\mathcal{J}_{\alpha, T}}+\left.\|v\|\right|_{\mathcal{J}_{\alpha, T}}\right)^{\nu-k-1-j}\|v\|_{\mathcal{J}_{\alpha, T}}^{j}\|w\|_{\mathcal{K}_{+}}^{k} \\
& \int_{s}^{t}(t-\tau)^{\frac{n}{2 l}-\frac{n(\nu-k)}{2 \alpha l}-\frac{n k}{2 q}-\frac{\sigma}{2}} \tau^{\left(\frac{n}{2 \alpha l}-\frac{n}{2 p_{0}}\right)(\nu-k-1)}\|u(\tau, \cdot)-v(\tau, \cdot)\|_{\mathcal{M}_{\alpha l, \alpha m}} d \tau \\
+ & C \sum_{j=0}^{\nu-1} C_{\nu, j}\left(\|u\|_{\mathcal{J}_{\alpha, T}}+\|v\|_{\mathcal{J}_{\alpha, T}}\right)^{\nu-j-1}\|v\|_{\mathcal{J}_{\alpha, T}}^{j} \\
& \int_{0}^{t}(t-\tau)^{\frac{n}{2 l}-\frac{n \nu}{2 \alpha l}-\frac{\sigma}{2}} \tau_{\frac{p_{0}}{\alpha l}-1}\|u(\tau, \cdot)-v(\tau, \cdot)\|_{\mathcal{M}_{\alpha l, \alpha m}} d \tau
\end{aligned}
$$

holds for all $u, v \in \mathcal{J}_{\alpha, T}$ and every $t \in(0, T)$, where $s=\max \{0, t-1\}$, $C_{\nu, k, j}=\left(\begin{array}{c}\nu \\ k\end{array}\right)\left(\begin{array}{c}\nu-k \\ j\end{array}\right)$ and $C_{\nu, j}=C_{\nu, 0, j}=\left(\begin{array}{c}\nu \\ j\end{array}\right)$. 
Proof. By definition, we have

$$
\Psi[u](\tau, \cdot)-\Psi[v](\tau, \cdot)=\mathbf{I}_{1}+\mathbf{I}_{2}+\mathbf{I}_{3},
$$

where

$$
\begin{aligned}
& \mathbf{I}_{1}=\sum_{k=1}^{\nu-1} \sum_{j=0}^{\nu-k-1} C_{\nu, k, j} \int_{0}^{s} \exp ((t-\tau) \Delta)\left\{(u(\tau, \cdot)-v(\tau, \cdot))^{\nu-k-j} v(\tau, \cdot)^{j} w(\tau, \cdot)^{k}\right\} d \tau, \\
& \mathbf{I}_{2}=\sum_{k=1}^{\nu-1} \sum_{j=0}^{\nu-k-1} C_{\nu, k, j} \int_{s}^{t} \exp ((t-\tau) \Delta)\left\{(u(\tau, \cdot)-v(\tau, \cdot))^{\nu-k-j} v(\tau, \cdot)^{j} w(\tau, \cdot)^{k}\right\} d \tau, \\
& \mathbf{I}_{3}=\sum_{j=0}^{\nu-1} C_{\nu, j} \int_{0}^{t} \exp ((t-\tau) \Delta)\left\{(u(\tau, \cdot)-v(\tau, \cdot))^{\nu-j} v(\tau, \cdot)^{j}\right\} d \tau,
\end{aligned}
$$

and $s=\max \{0, t-1\}, C_{\nu, k, j}=\left(\begin{array}{l}\nu \\ k\end{array}\right)\left(\begin{array}{c}\nu-k \\ j\end{array}\right), C_{\nu, j}=\left(\begin{array}{c}\nu \\ j\end{array}\right)$.

We first estimate $\mathbf{I}_{3}$. Since $u, v \in \mathcal{J}_{\alpha, T}$, it follows that

$$
\begin{aligned}
& \left.|| \mathbf{I}_{3}\right|_{\mathcal{N}_{l, m, 1}^{\sigma}} \\
& \leq \sum_{j=0}^{\nu-1} C_{\nu, j} \int_{0}^{t}\left\|\exp ((t-\tau) \Delta)\left\{(u(\tau, \cdot)-v(\tau, \cdot))^{\nu-j} v(\tau, \cdot)^{j}\right\}\right\|_{\mathcal{N}_{l, m, 1}^{\sigma}} d \tau \\
& \leq C \sum_{j=0}^{\nu-1} C_{\nu, j} \int_{0}^{t}(t-\tau)^{\frac{n}{2 l}-\frac{n \nu}{2 \alpha l}-\frac{\sigma}{2}}\left\|(u(\tau, \cdot)-v(\tau, \cdot))^{\nu-j} v(\tau, \cdot)^{j}\right\|_{\mathcal{N}_{l, m, \infty}^{\frac{n}{\alpha}-\frac{n \nu}{\alpha l}}} d \tau \\
& \leq C \sum_{j=0}^{\nu-1} C_{\nu, j} \int_{0}^{t}(t-\tau)^{\frac{n}{2 l}-\frac{n \nu}{2 \alpha l}-\frac{\sigma}{2}}\left\|(u(\tau, \cdot)-v(\tau, \cdot))^{\nu-j} v(\tau, \cdot)^{j}\right\|_{\mathcal{M}_{\alpha l / \nu, \alpha m / \nu}} d \tau \\
& \leq C \sum_{j=0}^{\nu-1} C_{\nu, j} \int_{0}^{t}(t-\tau)^{\frac{n}{2 l}-\frac{n \nu}{2 \alpha l}-\frac{\sigma}{2}}\|u(\tau, \cdot)-v(\tau, \cdot)\|_{\mathcal{M}_{\alpha l, \alpha m}}^{\nu-j}\|v(\tau, \cdot)\|_{\mathcal{M}_{\alpha l, \alpha m}}^{j} d \tau \\
& \leq C \sum_{j=0}^{\nu-1} C_{\nu, j}\left(\|u\|_{\mathcal{J}_{\alpha, T}}+\|v\|_{\mathcal{J}_{\alpha, T}}\right)^{\nu-j-1}\|v\|_{\mathcal{J}_{\alpha, T}}^{j} \\
& \int_{0}^{t}(t-\tau)^{\frac{n}{2 l}-\frac{n \nu}{2 \alpha l}-\frac{\sigma}{2}} \tau^{\frac{p_{0}}{\alpha l}-1}\|u(\tau, \cdot)-v(\tau, \cdot)\|_{\mathcal{M}_{\alpha l, \alpha m}} d \tau .
\end{aligned}
$$

Next we estimate $\mathbf{I}_{2}$. Observing that $w(t, \cdot) \in \mathcal{M}_{q, \frac{q r}{p}}$, we put

$$
\frac{1}{p_{2}}=\frac{\nu-k}{\alpha l}+\frac{k}{q}, \quad \frac{1}{q_{2}}=\frac{\nu-k}{\alpha m}+\frac{p k}{q r},
$$


then we have $p_{2}<l$ and

$$
\begin{aligned}
& \left\|\mathbf{I}_{2}\right\|_{\mathcal{N}_{l, m, 1}^{\sigma}} \\
& \leq \sum_{k=1}^{\nu-1} \sum_{j=0}^{\nu-k-1} C_{\nu, k, j} \int_{s}^{t} \| \exp ((t-\tau) \Delta) \\
& \left\{(u(\tau, \cdot)-v(\tau, \cdot))^{\nu-k-j} v(\tau, \cdot)^{j} w(\tau, \cdot)^{k}\right\} \|_{\mathcal{N}_{l, m, 1}^{\sigma}} d \tau \\
& \leq \sum_{k=1}^{\nu-1} \sum_{j=0}^{\nu-k-1} C_{\nu, k, j} \int_{s}^{t}(t-\tau)^{\frac{n}{2 l}-\frac{n(\nu-k)}{2 \alpha l}-\frac{n k}{2 q}-\frac{\sigma}{2}} \\
& \left\|(u(\tau, \cdot)-v(\tau, \cdot))^{\nu-k-j} v(\tau, \cdot)^{j} w(\tau, \cdot)^{k}\right\|_{\mathcal{N}_{l, m, \infty}^{\frac{n}{\tau}-\frac{n(\nu-k)}{\alpha l}-\frac{n k}{q}}} d \tau \\
& \leq \sum_{k=1}^{\nu-1} \sum_{j=0}^{\nu-k-1} C_{\nu, k, j} \int_{s}^{t}(t-\tau)^{\frac{n}{2 l}-\frac{n(\nu-k)}{2 \alpha l}-\frac{n k}{2 q}-\frac{\sigma}{2}} \\
& \left\|(u(\tau, \cdot)-v(\tau, \cdot))^{\nu-k-j} v(\tau, \cdot)^{j} w(\tau, \cdot)^{k}\right\|_{\mathcal{N}_{p_{2}, q_{2}, \infty}^{0}} d \tau \\
& \leq \sum_{k=1}^{\nu-1} \sum_{j=0}^{\nu-k-1} C_{\nu, k, j} \int_{s}^{t}(t-\tau)^{\frac{n}{2 l}-\frac{n(\nu-k)}{2 \alpha l}-\frac{n k}{2 q}-\frac{\sigma}{2}}
\end{aligned}
$$

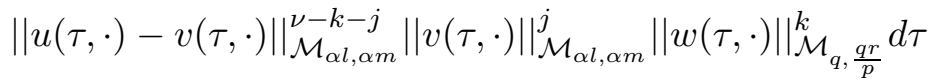

$$
\begin{aligned}
& \leq \sum_{k=1}^{\nu-1} \sum_{j=0}^{\nu-k-1} C_{\nu, k, j}\left(\|u\|_{\mathcal{J}_{\alpha, T}}+\|v\|_{\mathcal{J}_{\alpha, T}}\right)^{\nu-k-1-j}\|v\|_{\mathcal{J}_{\alpha, T}}^{j}\|w\|_{\mathcal{K}_{+}}^{k} \\
& \int_{S}^{t}(t-\tau)^{\frac{n}{2 l}-\frac{n(\nu-k)}{2 \alpha l}-\frac{n k}{2 q}-\frac{\sigma}{2}} \tau^{\left(\frac{n}{2 \alpha l}-\frac{n}{2 p_{0}}\right)(\nu-k-1)}\|u(\tau, \cdot)-v(\tau, \cdot)\|_{\mathcal{M}_{\alpha l, \alpha m}} d \tau,
\end{aligned}
$$

where $\frac{n}{2 l}-\frac{n(\nu-k)}{2 \alpha l}-\frac{n k}{2 q}-\frac{\sigma}{2}>-1,\left(\frac{n}{2 \alpha l}-\frac{n}{2 p_{0}}\right)(\nu-k)>-1$, for each $k=1,2, \ldots, \nu-1$.

We finally estimate $\mathbf{I}_{1}$. If $t \leq 1$, then we have $\mathbf{I}_{1}=0$. Suppose that $t>1$. Put

$$
\frac{1}{p_{1}}=\frac{\nu-k}{\alpha l}+\frac{k}{p}, \quad \frac{1}{q_{1}}=\frac{\nu-k}{\alpha m}+\frac{k}{r},
$$


then we have $p_{1}<l$ and

$$
\begin{aligned}
& || \mathbf{I}_{1} \|_{\mathcal{N}_{l, m, 1}^{\sigma}} \\
& \leq \sum_{k=1}^{\nu-1} \sum_{j=0}^{\nu-k-1} C_{\nu, k, j} \int_{0}^{s} \| \exp ((t-\tau) \Delta) \\
& \left\{(u(\tau, \cdot)-v(\tau, \cdot))^{\nu-k-j} v(\tau, \cdot)^{j} w(\tau, \cdot)^{k}\right\} \|_{\mathcal{N}_{l, m, 1}^{\sigma}} d \tau \\
& \leq \sum_{k=1}^{\nu-1} \sum_{j=0}^{\nu-k-1} C_{\nu, k, j} \int_{0}^{s}(t-\tau)^{\frac{n}{2 l}-\frac{n(\nu-k)}{2 \alpha l}-\frac{n k}{2 p}-\frac{\sigma}{2}} \\
& \left\|(u(\tau, \cdot)-v(\tau, \cdot))^{\nu-k-j} v(\tau, \cdot)^{j} w(\tau, \cdot)^{k}\right\|_{\mathcal{N}_{l, m, \infty}^{\frac{n}{\tau}-\frac{n(\nu-k)}{\alpha l}-\frac{n k}{p}}} d \tau \\
& \leq \sum_{k=1}^{\nu-1} \sum_{j=0}^{\nu-k-1} C_{\nu, k, j} \int_{0}^{s}(t-\tau)^{\frac{n}{2 l}-\frac{n(\nu-k)}{2 \alpha l}-\frac{n k}{2 p}-\frac{\sigma}{2}} \\
& \left\|(u(\tau, \cdot)-v(\tau, \cdot))^{\nu-k-j} v(\tau, \cdot)^{j} w(\tau, \cdot)^{k}\right\|_{\mathcal{N}_{p_{1}, q_{1}, \infty}^{0}} d \tau \\
& \leq \sum_{k=1}^{\nu-1} \sum_{j=0}^{\nu-k-1} C_{\nu, k, j} \int_{0}^{s}(t-\tau)^{\frac{n}{2 l}-\frac{n(\nu-k)}{2 \alpha l}-\frac{n k}{2 p}-\frac{\sigma}{2}} \\
& \|u(\tau, \cdot)-v(\tau, \cdot)\|_{\mathcal{M}_{\alpha l, \alpha m}-k-j}^{\nu}\|v(\tau, \cdot)\|_{\mathcal{M}_{\alpha l, \alpha m}}^{j}\|w(\tau, \cdot)\|_{\mathcal{M}_{p, r}}^{k} d \tau \\
& \leq \sum_{k=1}^{\nu-1} \sum_{j=0}^{\nu-k-1} C_{\nu, k, j}\left(\|u\|_{\mathcal{J}_{\alpha, T}}+\|v\|_{\mathcal{J}_{\alpha, T}}\right)^{\nu-k-1-j}\|v\|_{\mathcal{J}_{\alpha, T}}^{j}\|w\|_{\mathcal{K}_{+}}^{k} \\
& \int_{0}^{s}(t-\tau)^{\frac{n}{2 l}-\frac{n(\nu-k)}{2 \alpha l}-\frac{n k}{2 p}-\frac{\sigma}{2}} \tau^{\left(\frac{n}{2 \alpha l}-\frac{n}{2 p_{0}}\right)(\nu-k-1)}\|u(\tau, \cdot)-v(\tau, \cdot)\|_{\mathcal{M}_{\alpha l, \alpha m}} d \tau,
\end{aligned}
$$

This completes the proof.

Proposition 4.2. For every $\sigma$ such that $\frac{n}{l}-\frac{n \nu}{\alpha l}<\sigma \leq \frac{n}{l}-\frac{n}{\alpha l}$, there exists a positive constant $C$ independent of $w \in \mathcal{K}_{+}$and $T \in(0, \infty]$ such that the estimate

$$
\begin{gathered}
\sup _{0<t<T} t^{\frac{\sigma}{2}-\frac{n}{2 l}+\frac{n}{2 p_{0}}}\|\Psi[u]-\Psi[v]\|_{\mathcal{N}_{l, m, 1}^{\sigma}} \\
\leq C\|u-v\| \mathcal{J}_{\alpha, T} \sum_{k=0}^{\nu-1} \sum_{j=0}^{\nu-k-1} C_{\nu, k, j}\left(\|u\|_{\mathcal{J}_{\alpha, T}}+\|v\| \mathcal{J}_{\alpha, T}\right)^{\nu-k-1-j}\|v\|_{\mathcal{J}_{\alpha, T}}^{j}\|w\|_{\mathcal{K}_{+}}^{k}
\end{gathered}
$$

holds for every $u, v \in \mathcal{J}_{\alpha, T}$. 
Proof. It is enough to estimate the integrals in Proposition 4.1. Denote

$$
\begin{aligned}
\mathbf{I}_{1, k}^{\prime} & =\int_{0}^{s}(t-\tau)^{\frac{n}{2 l}-\frac{n(\nu-k)}{2 \alpha l}-\frac{n k}{2 p}-\frac{\sigma}{2}} \tau^{\left(\frac{n}{2 \alpha l}-\frac{n}{2 p_{0}}\right)(\nu-k-1)}\|u(\tau, \cdot)-v(\tau, \cdot)\|_{\mathcal{M}_{\alpha l, \alpha m}} d \tau, \\
\mathbf{I}_{2, k}^{\prime} & =\int_{s}^{t}(t-\tau)^{\frac{n}{2 l}-\frac{n(\nu-k)}{2 \alpha l}-\frac{n k}{2 q}-\frac{\sigma}{2}} \tau^{\left(\frac{n}{2 \alpha l}-\frac{n}{2 p_{0}}\right)(\nu-k-1)}\|u(\tau, \cdot)-v(\tau, \cdot)\|_{\mathcal{M}_{\alpha l, \alpha m}} d \tau, \\
\mathbf{I}_{3}^{\prime} & =\int_{0}^{t}(t-\tau)^{\frac{n}{2 l}-\frac{n \nu}{2 \alpha l}-\frac{\sigma}{2}} \tau^{\frac{p_{0}}{\alpha l}-1}\|u(\tau, \cdot)-v(\tau, \cdot)\|_{\mathcal{M}_{\alpha l, \alpha m}} d \tau
\end{aligned}
$$

where $k=1,2, \ldots, \nu-1$ and $s=\max \{0, t-1\}$.

For $\mathbf{I}_{3}^{\prime}$, since $\sigma \leq \frac{n}{l}-\frac{n}{\alpha l}$, we have $-1<\frac{n}{2 l}-\frac{n \nu}{2 \alpha l}-\frac{\sigma}{2}<0$ and $-1<$ $\frac{n \nu}{2 \alpha l}-\frac{n \nu}{2 p_{0}}<0$. It follows that

$$
\begin{aligned}
\mathbf{I}_{3}^{\prime} & \leq t^{\frac{n}{2 l}-\frac{n}{2 p_{0}}-\frac{\sigma}{2}}\|u-v\|_{\mathcal{J}_{\alpha, T}} \int_{0}^{1}(1-\theta)^{\frac{n}{2 l}-\frac{n \nu}{2 \alpha l}-\frac{\sigma}{2}} \theta^{\frac{n \nu}{2 \alpha l}-\frac{n \nu}{2 p_{0}}} d \theta \\
& \leq B\left(\frac{n}{2 l}-\frac{n \nu}{2 \alpha l}-\frac{\sigma}{2}+1, \frac{n \nu}{2 \alpha l}-\frac{n \nu}{2 p_{0}}+1\right) t^{\frac{n}{2 l}-\frac{n}{2 p_{0}}-\frac{\sigma}{2}}\|u-v\|_{\mathcal{J}_{\alpha, T}}
\end{aligned}
$$

We next estimate $\mathbf{I}_{1, k}^{\prime}(k=1,2, \ldots, \nu-1)$. If $t \leq 1$, then we have $\mathbf{I}_{1, k}^{\prime}=0$. Suppose that $t>1$. Then we have

$$
\mathbf{I}_{1, k}^{\prime} \leq\|u-v\|_{\mathcal{J}_{\alpha, T}} \int_{0}^{t-1}(t-\tau)^{\frac{n}{2 l}-\frac{n(\nu-k)}{2 \alpha l}-\frac{n k}{2 p}-\frac{\sigma}{2}} \tau^{\frac{n(\nu-k)}{2 \alpha l}-\frac{n(\nu-k)}{2 p_{0}}} d \tau .
$$

If $1<t \leq 2$, we have $t-1 \leq \frac{t}{2}$ and

$$
\begin{aligned}
& \int_{0}^{t-1}(t-\tau)^{\frac{n}{2 l}-\frac{n(\nu-k)}{2 \alpha l}-\frac{n k}{2 p}-\frac{\sigma}{2}} \tau^{\frac{n(\nu-k)}{2 \alpha l}-\frac{n(\nu-k)}{2 p_{0}}} d \tau \\
\leq & \int_{0}^{\frac{t}{2}}(t-\tau)^{\frac{n}{2 l}-\frac{n(\nu-k)}{2 \alpha l}-\frac{n k}{2 p}-\frac{\sigma}{2}} \tau^{\frac{n(\nu-k)}{2 \alpha l}-\frac{n(\nu-k)}{2 p_{0}}} d \tau .
\end{aligned}
$$

On the other hand, if $t>2$, then we can write

$$
\begin{aligned}
& \int_{0}^{t-1}(t-\tau)^{\frac{n}{2 l}-\frac{n(\nu-k)}{2 \alpha l}-\frac{n k}{2 p}-\frac{\sigma}{2}} \tau^{\frac{n(\nu-k)}{2 \alpha l}-\frac{n(\nu-k)}{2 p_{0}}} d \tau \\
= & \int_{0}^{\frac{t}{2}}(t-\tau)^{\frac{n}{2 l}-\frac{n(\nu-k)}{2 \alpha l}-\frac{n k}{2 p}-\frac{\sigma}{2}} \tau^{\frac{n(\nu-k)}{2 \alpha l}-\frac{n(\nu-k)}{2 p_{0}}} d \tau \\
& +\int_{\frac{t}{2}}^{t-1}(t-\tau)^{\frac{n}{2 l}-\frac{n(\nu-k)}{2 \alpha l}-\frac{n k}{2 p}-\frac{\sigma}{2}} \tau^{\frac{n(\nu-k)}{2 \alpha l}-\frac{n(\nu-k)}{2 p_{0}}} d \tau .
\end{aligned}
$$


Furthermore, observing that $t>1$ and $p<p_{0}$, we have

$$
\begin{aligned}
& \int_{0}^{\frac{t}{2}}(t-\tau)^{\frac{n}{2 l}-\frac{n(\nu-k)}{2 \alpha l}-\frac{n k}{2 p}-\frac{\sigma}{2}} \tau^{\frac{n(\nu-k)}{2 \alpha l}-\frac{n(\nu-k)}{2 p_{0}}} d \tau \\
\leq & C t^{\frac{n}{2 l}-\frac{n}{2 p_{0}}-\frac{\sigma}{2}+k\left(\frac{n}{2 p_{0}}-\frac{n}{2 p}\right)} \int_{0}^{\frac{1}{2}}(1-\theta)^{\frac{n}{2 l}-\frac{n(\nu-k)}{2 \alpha l}-\frac{n k}{2 p}-\frac{\sigma}{2}} \theta^{\frac{n(\nu-k)}{2 \alpha l}-\frac{n(\nu-k)}{2 p_{0}}} d \theta \\
\leq & C t^{\frac{n}{2 l}-\frac{n}{2 p_{0}}-\frac{\sigma}{2}} .
\end{aligned}
$$

If $t>2$, we can estimate

$$
\begin{aligned}
& \int_{\frac{t}{2}}^{t-1}(t-\tau)^{\frac{n}{2 l}-\frac{n(\nu-k)}{2 \alpha l}-\frac{n k}{2 p}-\frac{\sigma}{2}} \tau^{\frac{n(\nu-k)}{2 \alpha l}-\frac{n(\nu-k)}{2 p_{0}}} d \tau \\
\leq & C t^{\frac{n(\nu-k)}{2 \alpha l}-\frac{n(\nu-k)}{2 p_{0}}} \int_{1}^{\frac{t}{2}} \theta^{\frac{n}{2 l}-\frac{n(\nu-k)}{2 \alpha l}-\frac{n k}{2 p}-\frac{\sigma}{2}} d \theta \\
\leq & \begin{cases}C t^{\frac{n}{2 l}-\frac{n}{2 p_{0}}-\frac{\sigma}{2}+k\left(\frac{n}{2 p_{0}}-\frac{n}{2 p}\right)}, & \text { if } \frac{n}{2 l}-\frac{n(\nu-k)}{2 \alpha l}-\frac{n k}{2 p}-\frac{\sigma}{2}+1>0, \\
C t^{\frac{n}{2 l}-\frac{n}{2 p_{0}}-\frac{\sigma}{2}+k\left(\frac{n}{2 p_{0}}-\frac{n}{2 p}\right)} \ln t, & \text { if } \frac{n}{2 l}-\frac{n(\nu-k)}{2 \alpha l}-\frac{n k}{2 p}-\frac{\sigma}{2}+1=0, \\
C t^{\frac{n(\nu-k)}{2 \alpha l}-\frac{n(\nu-k)}{2 p_{0}}}, & \text { if } \frac{n}{2 l}-\frac{n(\nu-k)}{2 \alpha l}-\frac{n k}{2 p}-\frac{\sigma}{2}+1<0 .\end{cases}
\end{aligned}
$$

Observe that $t>1$ and $p<p_{0}$, so we have

$$
\begin{gathered}
t^{k\left(\frac{n}{2 p_{0}}-\frac{n}{2 p}\right)} \leq C, \quad t^{k\left(\frac{n}{2 p_{0}}-\frac{n}{2 p}\right)} \ln t \leq C, \\
t^{\frac{n(\nu-k)}{2 \alpha l}-\frac{n(\nu-k)}{2 p_{0}}} \leq t^{\frac{n}{2 \alpha l}-\frac{n}{2 p_{0}}} \leq t^{\frac{n}{2 l}-\frac{n}{2 p_{0}}-\frac{\sigma}{2}}, \quad k=1,2, \ldots, \nu-1 .
\end{gathered}
$$

Hence (4.6) implies that

$$
\int_{\frac{t}{2}}^{t-1}(t-\tau)^{\frac{n}{2 l}-\frac{n(\nu-k)}{2 \alpha l}-\frac{n k}{2 p}-\frac{\sigma}{2}} \tau^{\frac{n(\nu-k)}{2 \alpha l}-\frac{n(\nu-k)}{2 p_{0}}} d \tau \leq C t^{\frac{n}{2 l}-\frac{n}{2 p_{0}}-\frac{\sigma}{2}} .
$$

It follows from (4.2), (4.3), (4.4), (4.5) and (4.7) that

$$
\mathbf{I}_{1, k}^{\prime} \leq C t^{\frac{n}{2 l}-\frac{n}{2 p_{0}}-\frac{\sigma}{2}}\|u-v\|_{\mathcal{J}_{\alpha, T}, \quad k=1,2, \ldots, \nu-1 .}
$$

We finally estimate $\mathbf{I}_{2, k}^{\prime}(k=1,2, \ldots, \nu-1)$. We have

$$
\mathbf{I}_{2, k}^{\prime} \leq\|u-v\|_{\mathcal{J}_{\alpha, T}} \int_{s}^{t}(t-\tau)^{\frac{n}{2 l}-\frac{n(\nu-k)}{2 \alpha l}-\frac{n k}{2 q}-\frac{\sigma}{2}} \tau^{\frac{n(\nu-k)}{2 \alpha l}-\frac{n(\nu-k)}{2 p_{0}}} d \tau
$$


The assumptions $\sigma \leq \frac{n}{l}-\frac{n}{\alpha l}$ and $p>\frac{(\nu-1)^{2}}{\nu^{2}-\nu-1} p_{0}$ imply that

$$
-1<\frac{n}{2 l}-\frac{n(\nu-k)}{2 \alpha l}-\frac{n k}{2 q}-\frac{\sigma}{2}<0, \quad-1<\frac{n(\nu-k)}{2 \alpha l}-\frac{n(\nu-k)}{2 p_{0}}<0,
$$

for $k=1,2, \ldots, \nu-1$.

If $0<t \leq 2$, we have

$$
\begin{aligned}
& \int_{s}^{t}(t-\tau)^{\frac{n}{2 l}-\frac{n(\nu-k)}{2 \alpha l}-\frac{n k}{2 q}-\frac{\sigma}{2}} \tau^{\frac{n(\nu-k)}{2 \alpha l}-\frac{n(\nu-k)}{2 p_{0}}} d \tau \\
\leq & \int_{0}^{t}(t-\tau)^{\frac{n}{2 l}-\frac{n(\nu-k)}{2 \alpha l}-\frac{n k}{2 q}-\frac{\sigma}{2}} \tau^{\frac{n(\nu-k)}{2 \alpha l}-\frac{n(\nu-k)}{2 p_{0}}} d \tau \\
\leq & C t^{\frac{n}{2 l}-\frac{n}{2 p_{0}}-\frac{\sigma}{2}+k\left(\frac{n}{2 p_{0}}-\frac{n}{2 q}\right)} \leq C t^{\frac{n}{2 l}-\frac{n}{2 p_{0}}-\frac{\sigma}{2}} .
\end{aligned}
$$

If $t>2$, we have $t-1>\frac{t}{2}$. It follows that

$$
\begin{aligned}
& \int_{s}^{t}(t-\tau)^{\frac{n}{2 l}-\frac{n(\nu-k)}{2 \alpha l}-\frac{n k}{2 q}-\frac{\sigma}{2}} \tau^{\frac{n(\nu-k)}{2 \alpha l}-\frac{n(\nu-k)}{2 p_{0}}} d \tau \\
\leq & (t-1)^{\frac{n(\nu-k)}{2 \alpha l}-\frac{n(\nu-k)}{2 p_{0}}} \int_{t-1}^{t}(t-\tau)^{\frac{n}{2 l}-\frac{n(\nu-k)}{2 \alpha l}-\frac{n k}{2 q}-\frac{\sigma}{2}} d \tau \\
\leq & C\left(\frac{t}{2}\right)^{\frac{n(\nu-k)}{2 \alpha l}-\frac{n(\nu-k)}{2 p_{0}}} \leq C t^{\frac{n}{2 \alpha l}-\frac{n}{2 p_{0}}} \\
\leq & C t^{\frac{n}{2 l}-\frac{n}{2 p_{0}}-\frac{\sigma}{2}}
\end{aligned}
$$

It follows from (4.9), (4.10) and (4.11) that

$$
\mathbf{I}_{2, k}^{\prime} \leq C t^{\frac{n}{2 l}-\frac{n}{2 p_{0}}-\frac{\sigma}{2}}\|u-v\|_{\mathcal{J}_{\alpha, T}}, \quad k=1,2, \ldots, \nu-1 .
$$

Summing up (4.1), (4.8) and (4.12) we obtain the conclusion.

Corollary 4.1. If $v \in \mathcal{J}_{\alpha, T}$, then we have $\Psi[v] \in \mathcal{J}_{\alpha, T}$. Furthermore, there exists a positive constant $C_{\alpha, 3}$ independent of $w \in \mathcal{K}_{+}$and $T \in(0, \infty]$ such that the estimate

$$
\left.\|\Psi[u]-\Psi[v]\|\right|_{\mathcal{J}_{\alpha, T}} \leq C_{\alpha, 3}\left(\|w\|_{\mathcal{K}_{+}}+\|u\|_{\mathcal{J}_{\alpha, T}}+\|v\|_{\mathcal{J}_{\alpha, T}}\right)\|u-v\|_{\mathcal{J}_{\alpha, T}}
$$

holds for every $u, v \in \mathcal{J}_{\alpha, T}$ satisfying $\|u\|_{\mathcal{J}_{\alpha, T}}<1,\|v\|_{\mathcal{J}_{\alpha, T}}<1$. 
Proof. Putting $\sigma=\frac{n}{l}-\frac{n}{\alpha l}$ in Proposition 4.2, we have

$$
\begin{aligned}
& \quad \mid \Psi[u]-\Psi[v] \|_{\mathcal{M}_{\alpha l, \alpha m}} \\
\leq & C\|\Psi[u]-\Psi[v]\|_{\mathcal{N}_{\alpha l, \alpha m, 1}^{0}} \\
\leq & C\|\Psi[u]-\Psi[v]\| \|_{\mathcal{N}_{\mathcal{N}^{\prime}, 1}^{\frac{n}{\alpha}-\frac{n}{\alpha l}}} \\
\leq & C t^{\frac{n}{2 \alpha l}-\frac{n}{2 p_{0}}} \sum_{k=0}^{\nu-1} \sum_{j=0}^{\nu-k-1} C_{\nu, k, j}\left(\|u\|_{\mathcal{J}_{\alpha, T}}+\|v\|_{\mathcal{J}_{\alpha, T}}\right)^{\nu-k-1-j} \\
& \quad \times\|v\|_{\mathcal{J}_{\alpha, T}}^{j}\|w\|_{\mathcal{K}_{+}}^{k}\|u-v\|_{\mathcal{J}_{\alpha, T}} \\
\leq & C_{\alpha, 3} t^{\frac{n}{2 \alpha l}-\frac{n}{2 p_{0}}}\left(\|w\|_{\mathcal{K}_{+}}+\|u\|_{\mathcal{J}_{\alpha, T}}+\|v\|_{\mathcal{J}_{\alpha, T}}\right)\|u-v\|_{\mathcal{J}_{\alpha, T}} .
\end{aligned}
$$

This implies the conclusion.

Corollary 4.2. For every $\beta$ such that $1<\beta<\alpha$, there exists a positive constant $C_{\alpha, \beta}$ independent of $w \in \mathcal{K}_{+}$and $T \in(0, \infty]$ such that the estimate

$$
\left.\sup _{0<t<T} t^{\frac{n}{2 p_{0}}-\frac{n}{2 \beta l}}|| \Psi[v]\right|_{\mathcal{M}_{\beta l, \beta m}} \leq C_{\alpha, \beta}\left(\|w\|_{\mathcal{K}_{+}}+\|v\|_{\mathcal{J}_{\alpha, T}}\right)\|v\|_{\mathcal{J}_{\alpha, T}}
$$

holds for every $v \in \mathcal{J}_{\alpha, T}$ with $\|v\|_{\mathcal{J}_{\alpha, T}}<1$. In particular, we have $\Psi[v] \in$ $\mathcal{J}_{\beta, T}$.

Proof. This is proved in the same way as in Corollary 4.1.

Corollary 4.3. Suppose that $v \in \mathcal{J}_{\alpha, T}$ satisfying $\|v\|_{\mathcal{J}_{\alpha, T}}<1$. Then we have $\Psi[v](t, \cdot) \rightarrow 0$ weakly* in $\dot{B}_{\infty, \infty}^{-\frac{n}{p_{0}}}$ as $t \rightarrow+0$.

Proof. Let $a(x)$ be a function in $\dot{B}_{1,1}^{\frac{n}{p_{0}}}$ and let $\varepsilon$ be a positive number. Then there exists a function $\varphi(x) \in \mathcal{S}$ such that $\operatorname{supp} \mathcal{F}[\varphi](\xi)$ is a compact subset of $\mathbf{R}^{n}$ away from the origin and that $\|a-\varphi\|_{\dot{B}_{1,1}}<\varepsilon$. From Proposition 4.2 with $u=0$ and $\sigma=\frac{n}{l}-\frac{n}{p_{0}}$, it follows that

$$
\begin{aligned}
& |(a, \Psi[v](t, \cdot))-(\varphi, \Psi[v](t, \cdot))| \\
\leq & \|a-\varphi\|_{\dot{B}_{1,1}^{\frac{n}{p_{0}}}}\|\Psi[v](t, \cdot)\|_{\dot{B}_{\infty, \infty}^{-\frac{n}{p_{0}}}} \\
\leq & C \varepsilon\|\Psi[v](t, \cdot)\|_{\mathcal{N}_{l, m, 1}^{\frac{n}{l}-\frac{n}{p_{0}}}} \leq C(v) \varepsilon .
\end{aligned}
$$


Moreover, in view of Proposition 4.2 with $u=0$ and $\sigma=\sigma_{0} \in$ $\left(\frac{n}{l}-\frac{n \nu}{\alpha l}, \frac{n}{l}-\frac{n}{p_{0}}\right)$, we have

$$
\begin{aligned}
|(\varphi, \Psi[v](t, \cdot))| & \leq\|\varphi\|_{\dot{B}_{1,1}^{-\sigma_{0}+\frac{n}{l}}}\|\Psi[v](t, \cdot)\|_{\dot{B}_{\infty, \infty}^{\sigma_{0}-\frac{n}{l}}} \\
& \leq C\|\varphi\|_{\dot{B}_{1,1}^{-\sigma_{0}+\frac{n}{l}}}\|\Psi[v](t, \cdot)\|_{\mathcal{N}_{l, m, 1}^{\sigma_{0}}} \\
& \leq C(v) t^{\frac{n}{2 l}-\frac{n}{2 p_{0}}-\frac{\sigma_{0}}{2}} .
\end{aligned}
$$

This, together with (4.13), implies that

$$
\limsup _{t \rightarrow+0}|(a, \Psi[v](t, \cdot))| \leq C(v) \varepsilon .
$$

Since $\varepsilon>0$ is arbitrary, we obtain the conclusion.

Lemma 4.1. For every $b(x) \in \dot{B}_{\infty, \infty}^{-\frac{n}{p_{0}}}$, we have $\exp (t \Delta) b \rightarrow b$ weakly* in $\dot{B}_{\infty, \infty}^{-\frac{n}{p_{0}}}$ as $t \rightarrow+0$.

Proof. For every $\varphi \in \dot{B}_{1,1}^{\frac{n}{p_{0}}}$, the strong continuity of the semigroup $\exp (t \Delta)$ on the space $\dot{B}_{1,1}^{\frac{n}{p_{0}}}$ implies

$$
\begin{aligned}
|(\varphi, \exp (t \Delta) b-b)| & =|(\exp (t \Delta) \varphi-\varphi, b)| \\
& \leq|| \exp (t \Delta) \varphi-\varphi\left\|_{\dot{B}_{1,1} \frac{n}{p_{0}}}\right\| b \|_{\dot{B}_{\infty, \infty}^{-\frac{n}{p_{0}}}} \\
& \rightarrow 0 \quad \text { as } \quad t \rightarrow+0 .
\end{aligned}
$$

This implies the conclusion.

Now we prove the main results on the stability of time-global solutions. First we have the following theorem.

TheOrem 4.1. Let $0<T \leq \infty$ and $w \in \mathcal{K}_{+}$be a solution of (1.1) satisfying the estimate $\|w\|_{\mathcal{K}_{+}}<\frac{1}{2 C_{\alpha, 3}}$. Then for every $b(x) \in \mathcal{S}^{\prime}$, the solution $u(t, x) \in \mathcal{J}_{\alpha, T}$ of the equation

$$
u(t, \cdot)=\exp (t \Delta) b+\Psi[u](t, \cdot)
$$

satisfying the conditions

$$
\|u\|_{\mathcal{J}_{\alpha, T}}<1, \limsup _{T \rightarrow+0}\|u\|_{\mathcal{J}_{\alpha, T}}<\frac{1}{4 C_{\alpha, 3}}
$$

is unique. 
Proof. Assume that $u_{1}(t, x), u_{2}(t, x) \in \mathcal{J}_{\alpha, T}$ enjoy (4.15) and (4.16) with $u=u_{j}, j=1,2$. Let $u_{0}=u_{1}-u_{2}$, we have $u_{0}=\Psi\left[u_{1}\right]-\Psi\left[u_{2}\right]$. Form (4.16) we can take $T_{0}>0$ such that $\left\|u_{j}\right\|_{\mathcal{J}_{\alpha, T_{0}}}<\frac{1}{4 C_{\alpha, 3}}$ for $j=1,2$. Corollary 4.1 implies that

$$
\left\|u_{0}||_{\mathcal{J}_{\alpha, T_{0}}} \leq C_{\alpha, 3}\left(\|w\|_{\mathcal{K}_{+}}+\left\|u_{1}\right\|_{\mathcal{J}_{\alpha, T_{0}}}+\| u_{2}|| \mathcal{J}_{\mathcal{J}_{, T_{0}}}\right)\right\| u_{0} \|_{\mathcal{J}_{\alpha, T_{0}}}
$$

Moreover, it follows from the assumptions that

$$
C_{\alpha, 3}\left(\|w\|_{\mathcal{K}_{+}}+\left\|u_{1}\right\|_{\mathcal{J}_{\alpha, T_{0}}}+\left\|u_{2}\right\|_{\mathcal{J}_{\alpha, T_{0}}}\right)<1
$$

We have $\left\|u_{0}\right\|_{\mathcal{J}_{\alpha, T_{0}}}=0$, which implies that $u_{1} \equiv u_{2}$ on $\left(0, T_{0}\right) \times \mathbf{R}_{x}^{n}$.

Next, in view of Proposition 4.1 with $\sigma=\frac{n}{l}-\frac{n}{\alpha l}$ and the inclusion relation $\mathcal{N}_{l, m, 1}^{\frac{n}{l}-\frac{n}{\alpha l}} \subset \mathcal{N}_{\alpha l, \alpha m, 1}^{0} \subset \mathcal{M}_{\alpha l, \alpha m}$, we have

$$
\begin{aligned}
& \left\|u_{0}(t, \cdot)\right\|_{\mathcal{M}_{\alpha l, \alpha m}} \\
\leq & \sum_{k=1}^{\nu-1} C(k) \int_{0}^{t} F_{k}(t-\tau) \tau^{\left(\frac{n}{2 \alpha l}-\frac{n}{2 p_{0}}\right)(\nu-k-1)}\left\|u_{0}(\tau, \cdot)\right\|_{\mathcal{M}_{\alpha l, \alpha m}} d \tau \\
& +C \int_{0}^{t}(t-\tau)^{-\frac{p_{0}}{\alpha l}} \tau^{\frac{p_{0}}{\alpha l}-1}\left\|u_{0}(\tau, \cdot)\right\|_{\mathcal{M}_{\alpha l, \alpha m}} d \tau,
\end{aligned}
$$

where $F_{k}(\theta)=\min \left\{\theta^{-\frac{p_{0}}{\alpha l}+k\left(\frac{n}{2 \alpha l}-\frac{n}{2 p}\right)}, \theta^{-\frac{p_{0}}{\alpha l}+k\left(\frac{n}{2 \alpha l}-\frac{n}{2 q}\right)}\right\}(k=1,2, \ldots, \nu-1)$. If we take $\varepsilon>0$ so small that

$$
\left\{\sum_{k=1}^{\nu-1} C(k) T_{0}^{\left(\frac{n}{2 \alpha l}-\frac{n}{2 p_{0}}\right)(\nu-k-1)} \int_{0}^{\varepsilon} F_{k}(\theta) d \theta+C T_{0}^{\frac{p_{0}}{\alpha l}-1} \int_{0}^{\varepsilon} \theta^{-\frac{p_{0}}{\alpha l}} d \theta\right\} \leq \frac{1}{2}
$$

holds, then

$$
\begin{aligned}
\sum_{k=1}^{\nu-1} C(k) \int_{s}^{t} F_{k}(t- & \tau) \tau^{\left(\frac{n}{2 \alpha l}-\frac{n}{2 p_{0}}\right)(\nu-k-1)} d \tau \\
& +C \int_{s}^{t}(t-\tau)^{-\frac{p_{0}}{\alpha l} \tau^{\frac{p_{0}}{\alpha l}-1} d \tau \leq \frac{1}{2}}
\end{aligned}
$$

holds for every $s, t$ such that $T_{0} \leq s \leq t<s+\varepsilon \leq T$. 
Now suppose that $u_{0}(t, x) \equiv 0$ on $(0, s) \times \mathbf{R}_{x}^{n}$, for some $s \geq T_{0}$. Let $t \in[s, s+\varepsilon)$. Then

$$
\begin{aligned}
& \left\|u_{0}(t, \cdot)\right\|_{\mathcal{M}_{\alpha l, \alpha m}} \\
\leq & \sum_{k=1}^{\nu-1} C(k) \int_{s}^{t} F_{k}(t-\tau) \tau^{\left(\frac{n}{2 \alpha l}-\frac{n}{2 p_{0}}\right)(\nu-k-1)}\left\|u_{0}(\tau, \cdot)\right\|_{\mathcal{M}_{\alpha l, \alpha m}} d \tau \\
& +C \int_{s}^{t}(t-\tau)^{-\frac{p_{0}}{\alpha l}} \tau^{\frac{p_{0}}{\alpha l}-1}\left\|u_{0}(\tau, \cdot)\right\|_{\mathcal{M}_{\alpha l, \alpha m}} d \tau \\
\leq & \sup _{s \leq \tau<s+\varepsilon}\left\|u_{0}(\tau, \cdot)\right\|_{\mathcal{M}_{\alpha l, \alpha m}} \\
& \times\left(\sum_{k=1}^{\nu-1} C(k) \int_{s}^{t} F_{k}(t-\tau) \tau^{\left(\frac{n}{2 \alpha l}-\frac{n}{2 p_{0}}\right)(\nu-k-1)} d \tau+C \int_{s}^{t}(t-\tau)^{-\frac{p_{0}}{\alpha l}} \tau^{\frac{p_{0}}{\alpha l}-1} d \tau\right) \\
\leq & \frac{1}{2} \sup _{s \leq \tau<s+\varepsilon}\left\|u_{0}(\tau, \cdot)\right\|_{\mathcal{M}_{\alpha l, \alpha m} \cdot}
\end{aligned}
$$

Taking the supremum with respect to $t \in[s, s+\varepsilon)$, we obtain

$$
\sup _{s \leq \tau<s+\varepsilon}\left\|u_{0}(\tau, \cdot)\right\|_{\mathcal{M}_{\alpha l, \alpha m}}=0
$$

which together with the assumption yields that $u_{1}(t, x) \equiv u_{2}(t, x)$ on $(0, s+$ $\varepsilon) \times \mathbf{R}_{x}^{n}$. Starting at $t=T_{0}$ and repeating this process, we arrive at $t=T$ after finite steps, which implies that $u_{1}(t, x) \equiv u_{2}(t, x)$ on $(0, T) \times \mathbf{R}_{x}^{n}$.

Proposition 4.3. Let $u(t, \cdot) \in \mathcal{J}_{\alpha, T}$ be a solution of $(4.15)$. Then for every $\beta$ such that $1<\beta<\alpha, u(t, \cdot) \in \mathcal{J}_{\beta, T}$. Moreover, it is Höldercontinuous in $t \in(0, T)$ with values in $\mathcal{M}_{\beta l, \beta m}$.

Proof. Since $b(x) \in \mathcal{N}_{l, m, \infty}^{\frac{n}{l}-\frac{n}{p_{0}}} \subset \mathcal{N}_{\beta l, \beta m, \infty}^{\frac{n}{\beta l}-\frac{n}{p_{0}}}$, it follows that $\exp (t \Delta) b \in$ $\mathcal{J}_{\beta, T}$ for every $\beta \in(1, \alpha)$. Moreover, we can prove that $\Psi[u](t, \cdot) \in \mathcal{J}_{\beta, T}$ in the same way as in Corollary 4.2 without the restriction $\|u\|_{\mathcal{J}_{\alpha, T}}<1$. Thus we conclude that $u(t, \cdot) \in \mathcal{J}_{\beta, T}$ for every $\beta \in(1, \alpha)$.

We next prove the Hölder continuity. Suppose that $0<s<t \leq s+1$. From the definition, we have

$$
u(t, \cdot)-u(s, \cdot)=\mathbf{I}_{1}+\mathbf{I}_{2}+\mathbf{I}_{3},
$$


where

$$
\begin{aligned}
& \mathbf{I}_{1}=(\exp ((t-s) \Delta)-1) u(s, \cdot) \\
& \mathbf{I}_{2}=\int_{s}^{t} \exp ((t-\tau) \Delta) \sum_{k=1}^{\nu-1}\left(\begin{array}{l}
\nu \\
k
\end{array}\right) u(\tau, \cdot)^{\nu-k} w(\tau, \cdot)^{k} d \tau \\
& \mathbf{I}_{3}=\int_{s}^{t} \exp ((t-\tau) \Delta) u(\tau, \cdot)^{\nu} d \tau
\end{aligned}
$$

Put $\sigma=\frac{n}{l}-\frac{n}{\alpha l}$. Since $\mathcal{N}_{l, m, 1}^{\frac{n}{l}-\frac{n}{\alpha l}} \subset \mathcal{N}_{\beta l, \beta m, 1}^{\frac{n}{\beta l}-\frac{n}{\alpha l}}$, we have $u(t, \cdot) \in \mathcal{N}_{\beta l, \beta m, 1}^{\frac{n}{\beta l}-\frac{n}{\alpha l}}$ for every $t>0$. It follows that

$$
\begin{aligned}
\left\|\mathbf{I}_{1}\right\|_{\mathcal{M}_{\beta l, \beta m}} & \leq C \int_{s}^{t}\left\|\frac{d}{d \tau} \exp ((\tau-s) \Delta) u(s, \cdot)\right\|_{\mathcal{N}_{\beta l, \beta m, 1}^{0}} d \tau \\
& \leq C \int_{s}^{t}(\tau-s)^{-1+\frac{n}{2 \beta l}-\frac{n}{2 \alpha l}}\|u(s, \cdot)\|_{\mathcal{N}_{\beta l, \beta m, 1}^{\frac{n}{\beta l}-\frac{n}{\alpha l}}} d \tau \\
& \leq C(s)(t-s)^{\frac{n}{2 \beta l}-\frac{n}{2 \alpha l}}
\end{aligned}
$$

where $C(s)$ is a constant depending on $s>0$.

Next we consider $\mathbf{I}_{2}$. Since the condition $\left(\mathrm{H}_{2}\right)$ implies that $p_{0}<q$ and $\frac{1}{\alpha l}<\frac{1}{q}$, we can obtain the following estimate in the same way as in Proposition 4.1.

$$
\begin{aligned}
& \leq C \sum_{k=1}^{\nu-1} \int_{s}^{t}\left\|\exp ((t-\tau) \Delta)\left\{u(\tau, \cdot)^{\nu-k} w(\tau, \cdot)^{k}\right\}\right\|_{\mathcal{N}_{l, m, 1}^{\frac{n}{l}-\frac{n}{\beta l}} d \tau} \leq\left. C \sum_{k=1}^{\nu-1}\|u\|\right|_{\mathcal{J}_{\alpha, T}} ^{\nu-k}\|w\|_{\mathcal{K}_{+}}^{k} \int_{s}^{t}(t-\tau)^{-\frac{p_{0}}{\alpha l}+\frac{n k}{2 \alpha l}-\frac{n k}{2 q}+\frac{n}{2 \beta l}-\frac{n}{2 \alpha l} \tau^{\left(\frac{n}{2 \alpha l}-\frac{n}{2 p_{0}}\right)(\nu-k)} d \tau} \\
& \leq C^{\prime}(s) \sum_{k=1}^{\nu-1}(t-s)^{1-\frac{p_{0}}{\alpha l}+\frac{n k}{2 \alpha l}-\frac{n k}{2 q}+\frac{n}{2 \beta l}-\frac{n}{2 \alpha l}} \\
& \leq C^{\prime}(s) \sum_{k=1}^{\nu-1}(t-s)^{1-\frac{p_{0}}{\alpha l}+\left(\frac{n}{2 \alpha l}-\frac{n}{2 q}\right)(\nu-1)+\frac{n}{2 \beta l}-\frac{n}{2 \alpha l}} \\
& \leq C^{\prime}(s)(t-s)^{1-\frac{p_{0}}{q}+\frac{n}{2 \beta l}-\frac{n}{2 \alpha l}}
\end{aligned}
$$

where $C^{\prime}(s)$ is another constant depending on $s>0$. 
We finally estimate $\mathbf{I}_{3}$ in the same way as $\mathbf{I}_{2}$. We have

$$
\begin{aligned}
\left\|\mathbf{I}_{3}\right\|_{\mathcal{M}_{\beta l, \beta m}} & \leq C\|u\|_{\mathcal{J}_{\alpha, T}}^{\nu} \int_{s}^{t}(t-\tau)^{-\frac{p_{0}}{\alpha l}+\frac{n}{2 \beta l}-\frac{n}{2 \alpha l}} \tau^{\frac{n \nu}{2 \alpha l}-\frac{n \nu}{2 p_{0}}} d \tau \\
& \leq C^{\prime \prime}(s)(t-s)^{1-\frac{p_{0}}{\alpha l}+\frac{n}{2 \beta l}-\frac{n}{2 \alpha l}}
\end{aligned}
$$

where $C^{\prime \prime}(s)$ is another constant depending on $s>0$.

Thus the Hölder continuity follows from (4.19), (4.20) and (4.21).

Proof of Theorem 2.2. Let $\rho=\frac{1}{4 C_{\alpha, 3}}, u(t, x)$ be a solution of $(2.3)$ on $(0, T) \times \mathbf{R}_{x}^{n}$ enjoying the assumptions of Theorem 2.2 . For every $\delta, t_{0} \in(0, T)$ such that $\delta<t_{0}$, multiply $\exp \left(\left(t_{0}-t\right) \Delta\right)$ to both sides of $(2.3)$ and integrate on $\left[\delta, t_{0}\right]$. Then we have

$$
\begin{gathered}
u\left(t_{0}, \cdot\right)=\exp \left(\left(t_{0}-\delta\right) \Delta\right) u(\delta, \cdot) \\
\quad+\int_{\delta}^{t_{0}} \exp \left(\left(t_{0}-t\right) \Delta\right) \sum_{k=0}^{\nu-1} u(t, \cdot)^{\nu-k} w(t, \cdot)^{k} d t \\
=\exp \left(\left(t_{0}-\delta\right) \Delta\right) u(\delta, \cdot)+\Psi[u]\left(t_{0}, \cdot\right) \\
\quad-\exp \left(\left(t_{0}-\delta\right) \Delta\right) \Psi[u](\delta, \cdot) .
\end{gathered}
$$

Let $\varphi(x)$ be an arbitrary function in $\mathcal{S}$, then we have

$$
\begin{aligned}
& \left|\left(\varphi, \exp \left(\left(t_{0}-\delta\right) \Delta\right) u(\delta, \cdot)-\exp \left(t_{0} \Delta\right) b\right)\right| \\
\leq & \left|\left(\exp \left(\left(t_{0}-\delta\right) \Delta\right) \varphi-\exp \left(t_{0} \Delta\right) \varphi, u(\delta, \cdot)\right)\right| \\
& +\left|\left(\exp \left(t_{0} \Delta\right) \varphi, u(\delta, \cdot)-b\right)\right| \\
\leq & || \exp \left(\left(t_{0}-\delta\right) \Delta\right) \varphi-\left.\left.\exp \left(t_{0} \Delta\right) \varphi\right|_{\dot{B}_{1,1}^{\frac{n}{p_{0}}}}|| u(\delta, \cdot)\right|_{\dot{B}_{\infty, \infty}^{-\frac{n}{p_{0}}}} \\
& +\left|\left(\exp \left(t_{0} \Delta\right) \varphi, u(\delta, \cdot)-b\right)\right| .
\end{aligned}
$$

In view of the condition (2.6) and the strong continuity of the semigroup $\exp (t \Delta)$ on $\dot{B}_{1,1}^{\frac{n}{p_{0}}}$, together with the inclusion relation $\mathcal{N}_{l, m, \infty}^{\frac{n}{l}-\frac{n}{p_{0}}} \subset \dot{B}_{\infty, \infty}^{-\frac{n}{p_{0}}}$, we see that the first term of the right-hand side of (4.23) tends to 0 as $\delta \rightarrow+0$. On the other hand, the condition (2.8) implies that the second term of the right-hand side of (4.23) tends to 0 as $\delta \rightarrow+0$. It follows that the function $\exp \left(\left(t_{0}-\delta\right) \Delta\right) u(\delta, \cdot)$ tends to $\exp \left(t_{0} \Delta\right) b$ in the weak-* topology of $\mathcal{S}^{\prime}$ as $\delta \rightarrow+0$. This fact and Corollary 4.3 implies that the right-hand side of (4.22) tends to $\exp \left(t_{0} \Delta\right) b+\Psi[u]\left(t_{0}, \cdot\right)$ in $\mathcal{S}^{\prime}$ as $\delta \rightarrow+0$. This implies (4.15).

Now the conclusion immediately follows from Theorem 4.1 and Proposition 4.3. 
Now we prove Theorem 2.3. First we construct a solution of (4.15) by successive approximation, then show that it is the desired solution of (2.3).

Define the sequence of functions $\left\{u_{j}(t, x)\right\}_{j=0}^{\infty}$ inductively by

$$
\begin{aligned}
u_{0}(t, \cdot) & =\exp (t \Delta) b, \\
u_{j+1}(t, \cdot) & =u_{0}(t, \cdot)+\Psi\left[u_{j}\right](t, \cdot), j \geq 0 .
\end{aligned}
$$

Then we have the following lemma.

Lemma 4.2. There exists a positive constant $C_{\alpha, 0}$ such that

$$
\left\|u_{0}\right\|_{\mathcal{J}_{\alpha, \infty}} \leq C_{\alpha, 0}\|b\|_{\mathcal{N}_{l, m, \infty}^{\frac{n}{l}-\frac{n}{p_{0}}}}
$$

Proof. This is a direct consequence from the following estimate.

$$
\begin{aligned}
\left\|u_{0}(t, \cdot)\right\|_{\mathcal{M}_{\alpha l, \alpha m}} & \leq C\|\exp (t \Delta) b\|_{\mathcal{N}_{\alpha l, \alpha m, 1}^{0}} \\
& \leq C\|\exp (t \Delta) b\|_{\mathcal{N}_{l, m, 1}^{\frac{n}{l}-\frac{n}{\alpha l}}} \\
& \leq C t^{\frac{n}{2 \alpha l}-\frac{n}{2 p_{0}}}\|b\|_{\mathcal{N}_{l, m, \infty}^{\frac{n}{p_{0}}-\frac{n}{p^{0}}}}
\end{aligned}
$$

Corollary 4.1 immediately yields the following lemma.

Lemma 4.3. For every $j=1,2, \ldots$, we have $u_{j} \in \mathcal{J}_{\alpha, \infty}$ and

$$
\begin{aligned}
&\left.\left\|u_{1}-u_{0}\right\|\right|_{\mathcal{J}_{\alpha, \infty}} \leq C_{\alpha, 3}\left(\|w\|_{\mathcal{K}_{+}}+\left.\left\|u_{0}\right\|\right|_{\mathcal{J}_{\alpha, \infty}}\right)\left\|u_{0}\right\|_{\mathcal{J}_{\alpha, \infty}} \\
&\left\|u_{j+1}-u_{j}\right\|_{\mathcal{J}_{\alpha, \infty}} \\
& \leq C_{\alpha, 3}\left(\|w\|\left\|_{\mathcal{K}_{+}}+\right\| u_{j}\left\|\left.\right|_{\mathcal{J}_{\alpha, \infty}}+\right\| u_{j-1} \|\left.\right|_{\mathcal{J}_{\alpha, \infty}}\right)\left\|u_{j}-u_{j-1}\right\|_{\mathcal{J}_{\alpha, \infty}} .
\end{aligned}
$$

Corollary 4.4. For every $j=0,1, \ldots$, we have the inequalities

$$
\begin{aligned}
& \left\|u_{j+1}\right\|_{\mathcal{J}_{\alpha, \infty}} \\
\leq & \left\|u_{0}\right\|_{\mathcal{J}_{\alpha, \infty}}+\sum_{k=0}^{j}\left\|u_{k+1}-u_{k}\right\|_{\mathcal{J}_{\alpha, \infty}} \\
\leq & \left\|u_{0}\right\|_{\mathcal{J}_{\alpha, \infty}}+C_{\alpha, 3}\left(\left\|u_{0}\right\|_{\mathcal{J}_{\alpha, \infty}}+\sum_{k=0}^{j-1}\left\|u_{k+1}-u_{k}\right\|_{\mathcal{J}_{\alpha, \infty}}\right)^{2} \\
& +C_{\alpha, 3}\|w\|_{\mathcal{K}_{+}}\left(\left\|u_{0}\right\|_{\mathcal{J}_{\alpha, \infty}}+\sum_{k=0}^{j-1}\left\|u_{k+1}-u_{k}\right\|_{\mathcal{J}_{\alpha, \infty}}\right) .
\end{aligned}
$$


Proof. We proceed by induction on $j$. The assertion for $j=0$ immediately follows from Lemma 4.2 and (4.24). Assume that the conclusion holds for $j \geq 0$. Then (4.25) implies that

$$
\begin{aligned}
& \left\|u_{j+2}\right\|_{\mathcal{J}_{\alpha, \infty}} \\
& \leq\left\|u_{j+1}\right\|_{\mathcal{J}_{\alpha, \infty}}+\left\|u_{j+2}-u_{j+1}\right\|_{\mathcal{J}_{\alpha, \infty}} \\
& \leq\left\|u_{0}\right\|_{\mathcal{J}_{\alpha, \infty}}+\sum_{k=0}^{j}\left\|u_{k+1}-u_{k}\right\|_{\mathcal{J}_{\alpha, \infty}}+\left\|u_{j+2}-u_{j+1}\right\|_{\mathcal{J}_{\alpha, \infty}} \\
& \leq\left\|u_{0}\right\|_{\mathcal{J}_{\alpha, \infty}}+C_{\alpha, 3}\left(\left\|u_{0}\right\|_{\mathcal{J}_{\alpha, \infty}}+\sum_{k=0}^{j-1}\left\|u_{k+1}-u_{k}\right\|_{\mathcal{J}_{\alpha, \infty}}\right)^{2} \\
& +C_{\alpha, 3}\|w\|_{\mathcal{K}_{+}}\left(\left\|u_{0}\right\|_{\mathcal{J}_{\alpha, \infty}}+\sum_{k=0}^{j-1}\left\|u_{k+1}-u_{k}\right\|_{\mathcal{J}_{\alpha, \infty}}\right) \\
& +C_{\alpha, 3}\left(\|w\|_{\mathcal{K}_{+}}+\left\|u_{j+1}\right\|_{\mathcal{J}_{\alpha, \infty}}+\left\|u_{j}\right\|_{\mathcal{J}_{\alpha, \infty}}\right)\left\|u_{j+1}-u_{j}\right\|_{\mathcal{J}_{\alpha, \infty}} \\
& \leq\left\|u_{0}\right\|_{\mathcal{J}_{\alpha, \infty}}+C_{\alpha, 3}\left(\left\|u_{0}\right\|_{\mathcal{J}_{\alpha, \infty}}+\sum_{k=0}^{j-1}\left\|u_{k+1}-u_{k}\right\|_{\mathcal{J}_{\alpha, \infty}}\right)^{2} \\
& +C_{\alpha, 3}\|w\|_{\mathcal{K}_{+}}\left(\left\|u_{0}\right\|_{\mathcal{J}_{\alpha, \infty}}+\sum_{k=0}^{j-1}\left\|u_{k+1}-u_{k}\right\|_{\mathcal{J}_{\alpha, \infty}}\right) \\
& +C_{\alpha, 3}\left\|u_{j+1}-u_{j}\right\|_{\mathcal{J}_{\alpha, \infty}} \\
& \left(\|w\|_{\mathcal{K}_{+}}+2\left\|u_{0}\right\|_{\mathcal{J}_{\alpha, \infty}}+2 \sum_{k=0}^{j-1}\left\|u_{k+1}-u_{k}\right\|_{\mathcal{J}_{\alpha, \infty}}+\left\|u_{j+1}-u_{j}\right\|_{\mathcal{J}_{\alpha, \infty}}\right) \\
& \leq\left\|u_{0}\right\|_{\mathcal{J}_{\alpha, \infty}}+C_{\alpha, 3}\left(\left\|u_{0}\right\|_{\mathcal{J}_{\alpha, \infty}}+\sum_{k=0}^{j}\left\|u_{k+1}-u_{k}\right\|_{\mathcal{J}_{\alpha, \infty}}\right)^{2} \\
& +C_{\alpha, 3}\|w\|_{\mathcal{K}_{+}}\left(\left\|u_{0}\right\|_{\mathcal{J}_{\alpha, \infty}}+\sum_{k=0}^{j}\left\|u_{k+1}-u_{k}\right\|_{\mathcal{J}_{\alpha, \infty}}\right) .
\end{aligned}
$$

which implies the conclusion with $j$ replace by $j+1$.

Remark 4.1. If $\|w\|_{\mathcal{K}_{+}}<\frac{1}{2 C_{\alpha, 3}}$ and $\|b\|_{\mathcal{N}_{l, m, \infty}^{\frac{n}{p_{0}}}}<\frac{1}{16 C_{\alpha, 0} C_{\alpha, 3}}$, then 
(4.26) implies the estimate

$$
\begin{aligned}
& \left\|u_{0}\right\|_{\mathcal{J}_{\alpha, \infty}}+\sum_{k=0}^{j}\left\|u_{k+1}-u_{k}\right\|_{\mathcal{J}_{\alpha, \infty}} \\
& \leq \omega_{\alpha}\left(\|b\|_{\mathcal{N}_{l, m, \infty}^{\frac{n}{l}-\frac{n}{p_{0}}}}\right)=\frac{1-\sqrt{1-16 C_{\alpha, 0} C_{\alpha, 3}\|b\|_{\mathcal{N}_{l, m, \infty}^{\frac{n}{l}-\frac{n}{p_{0}}}}}}{4 C_{\alpha, 3}},
\end{aligned}
$$

for every $j \geq 0$, which implies that $\left\|u_{j}\right\|_{\mathcal{J}_{\alpha, \infty}} \leq \frac{1}{4 C_{\alpha, 3}}<1$.

THEOREM 4.2. Suppose that $w \in \mathcal{K}_{+}$with $\|w\|_{\mathcal{K}_{+}}<\frac{1}{2 C_{\alpha, 3}}$ and $b(x) \in$ $\mathcal{N}_{l, m, \infty}^{\frac{n}{l}-\frac{n}{p_{0}}}$ satisfying the estimate

$$
\|b\|_{\mathcal{N}_{l, m, \infty}^{\frac{n}{l}-\frac{n}{p_{0}}}}<\frac{1}{16 C_{\alpha, 0} C_{\alpha, 3}} .
$$

Then there exists a solution $u(t, x) \in \mathcal{J}_{\alpha, \infty}$ of the equation (4.15) satisfying the estimate

$$
\|u\|_{\mathcal{J}_{\alpha, \infty}} \leq \omega_{\alpha}\left(\|b\|_{\mathcal{N}_{l, m, \infty}^{\frac{n}{l}-\frac{n}{p_{0}}}}\right)=\frac{1-\sqrt{1-16 C_{\alpha, 0} C_{\alpha, 3}\|b\|_{\mathcal{N}_{l, m, \infty}^{\frac{n}{l}-\frac{n}{p_{0}}}}}}{4 C_{\alpha, 3}}
$$

Moreover, the function $u(t, \cdot)$ enjoys $(2.9)$, (2.10) with a suitable function $\omega(\iota)$, and (2.11).

Proof. Remark 4.1 imples that $u_{j}(t, \cdot)$ converges in $\mathcal{M}_{\alpha l, \alpha m}$ as $j \rightarrow \infty$ for almost every $t>0$. Hence there exists a function $u(t, x) \in \mathcal{J}_{\alpha, \infty}$ such that $\lim _{j \rightarrow \infty} u_{j}(t, \cdot)=u(t, x)$ for almost every $t>0$. Moreover, we have (4.29). From this fact and the definition of $u_{j}$ we conclude that $u(t, x)$ satisfies (4.15). This completes the proof of the existence.

The fact (2.9) follows directly from Lemma 4.1 and Corollary 4.3.

Next we prove (2.11). It is easy to obtain the following estimate in the same way as in Lemma 4.2 .

$$
\left.\|\exp (t \Delta) b\|\right|_{\mathcal{N}_{l, m, \infty}^{\frac{n}{\tau}-\frac{n}{p_{0}}}} \leq C\left(\|b\|_{\mathcal{N}_{l, m, \infty}^{\frac{n}{l}-\frac{n}{p_{0}}}}\right)
$$




$$
\begin{aligned}
& \left\|\exp (t \Delta) b||_{\mathcal{M}_{\beta l, \beta m}} \leq C\right\| \exp (t \Delta) b \|_{\mathcal{N}_{\beta l, \beta m, 1}^{0}} \\
& \leq C\|\exp (t \Delta) b\|_{\mathcal{N}_{l, m, 1}^{\frac{n}{l}-\frac{n}{\beta l}}} \\
& \leq C t^{\frac{n}{2 \beta l}-\frac{n}{2 p_{0}}}\|b\|_{\mathcal{N}_{l, m, \infty}^{\frac{n}{l}-\frac{n}{p_{0}}}}
\end{aligned}
$$

for every $\beta$ such that $1<\beta<\alpha$. This implies that $\exp (t \Delta) b \in \mathcal{J}_{\beta, \infty}$.

The inequality (4.31) and Corollary 4.2 yields (2.11) for every $\beta \in$ $(1, \alpha)$.

Finally, in view of the fact $u \in \mathcal{J}_{\alpha, T}$, we can apply Proposition 4.2 with $\sigma=\frac{n}{l}-\frac{n}{p_{0}}$ to obtain

$$
\sup _{t>0}\|\Psi[u](t, \cdot)\|_{\mathcal{N}_{l, m, 1}^{\frac{n}{l}-\frac{n}{p_{0}}}} \leq C \omega\left(\|b\|_{\mathcal{N}_{l, m, \infty}^{\frac{n}{l}-\frac{n}{p_{0}}}}\right)
$$

with some constant $C$. This fact and (4.30), together with the equality (4.15), yield (2.10).

Proof of Theorem 2.3. In view of Theorem 4.2, the solution of (4.15) constructed above satisfies the assumptions of Theorem 2.2 and (2.9), (2.10) and (2.11). Moreover, the functions $w(t, x)$ and $u(t, x)$ are Hölder continuous with respect to $t \in(0, \infty)$, with values in the spaces $\mathcal{M}_{p, r} \cap \mathcal{M}_{q, \frac{q r}{p}}$ and $\mathcal{M}_{\beta l, \beta m}$ (for every $\beta$ such that $1<\beta<\alpha$ ) respectively. Now choose $\beta$ such that $\frac{\nu}{\alpha}<\frac{\nu}{\beta}<m$, then the function $u(t, \cdot)^{\nu-k} w(t, \cdot)^{k}$ is Hölder-continuous in $t>0$ with values in $\mathcal{N}_{p_{0}, r, \infty}^{s_{k}}$, where

$$
s_{k}=-\frac{n(k-1)}{p_{0}}-\frac{n(\nu-k)}{\beta l},
$$

for every $k=0,1, \ldots, \nu-1$. Then we can prove that the solution $u(t, x)$ of (4.15) also solves (2.3) in the same way as in Proposition 3.2.

Acknowledgements. The author expresses his deep gratitude to Professor Wei Jun-cheng for the hospitality during his visit to Department of Mathematics, the Chinese University of Hong Kong.

\section{REFERENCES}

[1] P. Baras, M. Pierre, Problèmes paraboliques semi-linéaires avec données measures, Applicable Anal., 18 (1984), 111-149. 
[2] H. Brezis, A. Friedman, Nonlinear parabolic equations involving measures as initial conditions, J. Math. Pure. Appl. (9), 62 (1983), 73-97.

[3] H. Fujita, On the blowing up of solutions of the Cauchy problem for $u_{t}=\Delta u+u^{1+\alpha}$, J. Fac. Sci. Univ. Tokyo, I, 13 (1966), 109-124.

[4] A. Haraux and F.B. Weissler, Non-uniqueness for a semilinear initial value problem, Indiana Univ. Math. J., 31 (1982), 167-189.

[5] K. Hayakawa, On nonexistence of global solutions of some semilinear parabolic equations, Proc. Japan Acad. A, 49 (1973), 503-505.

[6] K. Kobayashi, T. Sirao and H. Tanaka, On the growing up problem for semilinear heat equations, J. Math. Soc. Japan, 29 (1977), 407-424.

[7] H. Kozono,and M. Yamazaki, Semilinear heat equations and the Navier-Stokes equation with distributions as initial data, C. R. Acad. Sci. Paris, Sér. I, 317 (1993), $1127-1132$.

[8] Semilinear heat equations and the Navier-Stokes equation with distributions in new function spaces as initial data, Comm. in P.D.E., 19 (1994), 959-1014.

[9] — The stability of small stationary solutions in Morrey spaces of the Navier-Stokes equation, Indiana Univ. Math. J., 44, No.3 (1995), 1307-1336.

[10] Small stable stationary solutions in Morrey spaces of the Navier-Stokes equation, Proc. Japan Acad. Ser.A, 71 (1995), 199-201.

[11] T.-Y. Lee, Some limit theorems for super-Brownian motion and semilinear differential equations, Annals of Probability, 21 (1993), 979-995.

[12] Y. Niwa, Semilinear heat equations with measures as initial data, Thesis, Univ. of Tokyo, 1986.

[13] C.V. Pao, Periodic solutions of systems of parabolic equations in unbounded domains, Nonlinear Analysis, 40 (2000), 523-535.

[14] J. Peetre, On convolution operators leaving $L^{p, \lambda}$ spaces invariant, Ann. Mat. Pura Appl., 72 (1966), 295-304.

[15] M.E. Taylor, Analysis on Morrey spaces and applications to Navier-Stokes and other evolution equations, Comm. in P.D.E., 17 (1992), 1407-1456.

[16] F.B. Weissler, Existence and non-existence of global solutions for a semilinear heat equation, Israel J. Math., 38 (1981), 29-40.

[17] J. Wu, Well-posedness of a semilinear heat equation with weak initial data, J. Fourier Anal. Appl., 4 (1998), 629-642.

[18] M. Yamazaki, Solutions in the Morrey spaces of the Navier-Stokes equation with time-dependent external force, Funkcial. Ekvac., 43 (2000), No.3, 419-460.

[19] X.F. Zhou, The stability of small stationary solutions in Morrey spaces of the semilinear heat equations, J. Math. Sci. Univ. Tokyo, 6 (1999), 793-822.

Department of Mathematics

Wuhan University

Wuhan 430072, Hubei

P.R.China

xfzhou2003@hotmail.com 